\title{
Reflected backward SDEs with two barriers under monotonicity and general increasing conditions
}

\author{
Mingyu $\mathrm{Xu}$ * \\ Departement des Mathématiques, Université du Maine, Le Mans France; \\ Department of Financial Mathematics and Control science, School of Mathematical Science \\ Fudan University, Shanghai, 200433, China.
}

\begin{abstract}
In this paper, we prove the existence and uniqueness result of the reflected BSDE with two continuous barriers under monotonicity and general increasing condition on $y$, with Lipschitz condition on $z$.

Keywords: Reflected backward stochastic differential equation, monotonicity condition, comparison theorem, Dynkin game.
\end{abstract}

\section{Introduction}

Nonlinear backward stochastic differential equations (BSDEs in short) were firstly introduced by Pardoux and Peng (1990), who proved the existence and uniqueness of adapted solutions, when the coefficient $f$ is Lipschitz in $(y, z)$ uniformly in $(t, \omega)$, with square-integrability assumptions on the coefficient $f(t, \omega, y, z)$ and terminal condition $\xi$. Later, Pardoux (1999) and Briand, Delyon, $\mathrm{Hu}$, Pardoux and Stoica (2003) studied the solution of a BSDE with a coefficient $f(t, \omega, y, z)$ that satisfies only monotonicity, continuity and general increasing growth conditions with respect to $y$, and Lipschitz on $z$. That is, for some real number $\mu \in \mathbb{R}, k \geq 0$ and some continuous increasing function $\varphi: \mathbb{R}_{+} \rightarrow \mathbb{R}_{+}: \forall t \in[0, T], y, y^{\prime} \in \mathbb{R}, z, z^{\prime} \in \mathbb{R}^{d}$ :

$$
\begin{aligned}
|f(t, y, z)| & \leq|f(t, 0, z)|+\varphi(|y|), \\
\left(y-y^{\prime}\right)\left(f(t, y, z)-f\left(t, y^{\prime}, z\right)\right) & \leq \mu\left|y-y^{\prime}\right|^{2} \\
\left|f(t, y, z)-f\left(t, y, z^{\prime}\right)\right| & \leq k\left|z-z^{\prime}\right| .
\end{aligned}
$$

Reflected backward stochastic differential equations (RBSDEs in short) with one lower barrier were studied by El Karoui, Kapoudjian, Pardoux, Peng and Quenez (1997), in one dimension. The solution is constrained to remain above a continuous lower-boundary process with the help of an continuous increasing process. Later, Cvitanic and Karatzas (1996) studied the backward stochastic differential equation with two barriers. A solution to such equation associated to a terminal condition $\xi$, a coefficient $f(t, \omega, y, z)$ and two barriers $L$ and $U$, is a triple $(Y, Z, K)$ of adapted processes, valued in $\mathbb{R}^{1+d+1}$, which satisfies

$$
Y_{t}=\xi+\int_{t}^{T} f\left(s, Y_{s}, Z_{s}\right) d s+K_{T}^{+}-K_{t}^{+}-\left(K_{T}^{-}-K_{t}^{-}\right)-\int_{t}^{T} Z_{s} d B_{s}, 0 \leq t \leq T \text { a.s. }
$$

$L_{t} \leq Y_{t} \leq U_{t}, 0 \leq t \leq T$ and $\int_{0}^{T}\left(Y_{s}-L_{s}\right) d K_{s}^{+}=\int_{0}^{T}\left(Y_{s}-U_{s}\right) d K_{s}^{-}=0$, a.s. In this case, a solution $Y$ has to remain between the lower boundary $L$ and upper boundary $U$, almost surely. This is

*Email: xvmingyu@gmail.com 
achieved by the cumulative action of two continuous, increasing reflecting processes $K^{ \pm}$, which act in a minimal way when $Y$ attempts to cross barriers. And the authors proved the existence and uniqueness of the solution when $f(t, \omega, y, z)$ is Lipschitz on $(y, z)$ uniformly in $(t, \omega)$ and when $L<U$ on $[0, T]$ and there exists a different supermartingale between $L$ and $U$ (Mokobodski's assumption in Dynkin game). Furthermore they established the connection between solution $Y$ and the value of Dynkin games (certain stochastic games of stopping). Then in [8, the existence of a solution was proved when $f$ is only continuous with linear growth in $(y, z)$, but in the case when one obstacle is smooth. Later, Lepeltier and San Martin used the penalization method to prove the existence of a solution to such equation, with same assumption on $f$ as in [8], without extra smoothness of the barriers, i.e. when $L$ and $U$ are continuous, $L<U$ on $[0, T]$, and Mokobodski's assumption.

More recently, Lepeltier, Matoussi and Xu proved the existence and uniqueness of the solution to the reflected BSDE with one lower continuous barrier under the assumption (11) for $f$. The existence is proved by approximation. In this paper, we consider the reflected BSDE with two continuous barrier under the assumption (11), and give the uniqueness and existence of the solution, which is obtained by approximation.

The paper is organized as following: In subsection 2.1, we present notations and assumptions; then we prove the main results of this paper, the existence and uniqueness of the solution in subsection 2.2; in subsection 2.3 we prove an important theorem for the existence in five steps. Finally, in section 3, we prove several comparison theorems with respect to RBSDE with one or two barriers, which are used in the proof of existence.

\section{RBSDE's with two continuous barriers}

\subsection{Assumptions and notations}

Let $(\Omega, \mathcal{F}, P)$ be a complete probability space, and $B=\left(B_{1}, B_{2}, \cdots, B_{d}\right)^{\prime}$ be a $d$-dimensional Brownian motion defined on a finite interval $[0, T], 0<T<+\infty$. Denote by $\left\{\mathcal{F}_{t} ; 0 \leq t \leq T\right\}$ the natural filtration generated by the Brownian motion $B$ :

$$
\mathcal{F}_{t}=\sigma\left\{B_{s} ; 0 \leq s \leq t\right\},
$$

where $\mathcal{F}_{0}$ contains all $P$-null sets of $\mathcal{F}$.

We denote the following notations. For any given $n \in \mathbf{N}$, let us introduce the following spaces:

$$
\begin{aligned}
& \mathbf{L}_{n}^{2}\left(\mathcal{F}_{t}\right)=\left\{\xi: n \text {-dimensional } \mathcal{F}_{t} \text {-measurable random variable, s.t. } E\left(|\xi|^{2}\right)<+\infty\right\}, \\
& \mathbf{H}_{n}^{2}(0, T)=\left\{\psi: n \text {-dimensional } \mathcal{F}_{t} \text {-predictable process on the interval }[0, T]\right. \text {, } \\
& \text { s.t. } \left.E \int_{0}^{T}\|\psi(t)\|^{2} d t<+\infty\right\} \text {, } \\
& \mathbf{S}_{n}^{2}(0, T)=\left\{\psi: n \text {-dimensional } \mathcal{F}_{t}\right. \text {-progressively measurable continuous process } \\
& \text { on the interval } \left.[0, T] \text {, s.t. } E\left(\sup _{0 \leq t \leq T}\|\psi(t)\|^{2}\right)<+\infty\right\} \text {, } \\
& \mathbf{A}^{2}(0, T)=\left\{K \text { : real valued } \mathcal{F}_{t} \text {-adapted increasing continuous process, s.t. } K(0)=0\right. \text {, } \\
& \text { and } \left.E\left(K(T)^{2}\right)<+\infty\right\} \text {. } \\
& \mathbf{V F}^{2}(0, T)=\left\{V \text { : real valued } \mathcal{F}_{t^{-}}\right. \text {adapted continuous process with finite variation, s.t. } \\
& \left.V=K^{+}-K^{-} \text {, with } K^{ \pm} \in \mathbf{A}^{2}(0, T)\right\} \text {. }
\end{aligned}
$$

Finally, we shall denote by $\mathcal{P}$ the $\sigma$-algebra of predictable sets on $[0, T] \times \Omega$. In the real-valued case, i.e., $n=1$, these spaces will be simply denoted by $\mathbf{L}^{2}\left(\mathcal{F}_{t}\right), \mathbf{H}^{2}(0, T)$ and $\mathbf{S}^{2}(0, T)$, respectively.

Let us consider the reflected backward stochastic differential equation with monotonic condition in $y$ on a fixed time interval; we need the following assumptions: 
Assumption 2.1. A final condition $\xi \in \mathbf{L}^{2}\left(\mathcal{F}_{T}\right)$.

Assumption 2.2. A coefficient $f: \Omega \times[0, T] \times \mathbf{R} \times \mathbf{R}^{d} \rightarrow \mathbb{R}$, satisfying for some continuous increasing function $\varphi: \mathbb{R}_{+} \rightarrow \mathbb{R}_{+}$, real numbers $\mu \in \mathbb{R}$ and $k>0$ :

(i) $\quad f(\cdot, y, z)$ is progressively measurable, $\forall(y, z) \in \mathbb{R} \times \mathbb{R}^{d}$;

(ii) $|f(t, y, z)| \leq|f(t, 0, z)|+\varphi(|y|), \forall(t, y, z) \in[0, T] \times \mathbb{R} \times \mathbb{R}^{d}$, a.s.;

(iii) $E \int_{0}^{T}|f(t, 0,0)|^{2} d t<\infty$;

(iv) $\left|f(t, y, z)-f\left(t, y, z^{\prime}\right)\right| \leq k\left|z-z^{\prime}\right|, \forall(t, y) \in[0, T] \times \mathbb{R}, z, z^{\prime} \in \mathbb{R}^{d}$, a.s.

(v) $\left(y-y^{\prime}\right)\left(f(t, y, z)-f\left(t, y^{\prime}, z\right)\right) \leq \mu\left|y-y^{\prime}\right|^{2}, \forall(t, z) \in[0, T] \times \mathbb{R}^{d}, y, y^{\prime} \in \mathbb{R}$, a.s.

(vi) $y \rightarrow f(t, y, z)$ is continuous, $\forall(t, z) \in[0, T] \times \mathbb{R}^{d}$, a.s.

Assumption 2.3. Two barriers $L_{t}, U_{t}$, which are $\mathcal{F}_{t}$-progressively measurable continuous processes, defined on the interval $[0, T]$, satisfying

(i)

$$
E\left[\varphi^{2}\left(\sup _{0 \leq t \leq T}\left(e^{\mu t}\left(L_{t}\right)^{+}\right)\right)\right]<\infty, E\left[\varphi^{2}\left(\sup _{0 \leq t \leq T}\left(e^{\mu t}\left(U_{t}\right)^{-}\right)\right)\right]<\infty
$$

$(L)^{+},(U)^{-} \in \mathbf{S}^{2}(0, T)$, and $L_{T} \leq \xi \leq U_{T}$, a.s., where $(L)^{+}$(resp. $\left.(U)^{-}\right)$is the positive part (resp. negative) part of $L$ (resp. $U$ ).

(ii) there exists a process $J_{t}=J_{0}+\int_{0}^{t} \phi_{s} d B_{s}-V_{t}^{+}+V_{t}^{-}, J_{T}=\xi$ with $\phi \in \mathbf{H}_{d}^{2}(0, T), V^{+}, V^{-} \in$ $\mathbf{A}^{2}(0, T)$, s.t.

$$
L_{t} \leq J_{t} \leq U_{t}, \text { for } 0 \leq t \leq T .
$$

(iii) $L_{t}<U_{t}$, a.s., for $0 \leq t<T$.

Now we introduce the definition of the solution of RBSDE with two barriers $L$ and $U$.

Definition 2.1 We say that $\left(Y_{t}, Z_{t}, K_{t}\right)_{0 \leq t \leq T}$ is a solution of the backward stochastic differential equation with two continuous reflecting barriers $L(\cdot)$ and $U(\cdot)$, terminal condition $\xi$ and coefficient $f$, which is denoted as $R B S D E(\xi, f, L, U)$, if the followings hold:

(1) $Y \in \mathbf{S}^{2}(0, T), Z \in \mathbf{H}_{d}^{2}(0, T)$, and $K \in \mathbf{V F}^{2}(0, T), K=K^{+}-K^{-}$, where $K^{ \pm} \in \mathbf{A}^{2}(0, T)$.

(2) $Y_{t}=\xi+\int_{t}^{T} f\left(s, Y_{s}, Z_{s}\right) d s+K_{T}^{+}-K_{t}^{+}-\left(K_{T}^{-}-K_{t}^{-}\right)-\int_{t}^{T} Z_{s} d B_{s}, \quad 0 \leq t \leq T$ a.s.

(3) $L_{t} \leq Y_{t} \leq U_{t}, 0 \leq t \leq T$, a.s.

(4) $\int_{0}^{T}\left(Y_{s}-L_{s}\right) d K_{s}^{+}=\int_{0}^{T}\left(Y_{s}-U_{s}\right) d K_{s}^{-}=0$, a.s.

Actually, a general solution of our $\operatorname{RBSDE}(\xi, f, L, U)$ would satisfy the assumptions (1) to (4). The state-process $Y(\cdot)$ is forced to stay between the barrier $L(\cdot)$ and $U(\cdot)$, thanks to the cumulation action of the reflection processes $K^{+}(\cdot)$ and $K^{-}(\cdot)$ respectively, which act only when necessary to prevent $Y(\cdot)$ from crossing the respective barrier, and in this sense, its action can be considered minimal, i.e. the integrability assumption (4). From the fact that $K^{ \pm} \in \mathbf{A}^{2}(0, T)$ is continuous and (2), it follows that $Y$ is continuous.

Remark 2.1 We have an analogue result of Proposition 4.1 in [4]. Precisely, the square-integrable solution $Y$ of the $R B S D E(\xi, f, L, U)$ is the value of the Dynkin game problem, whose payoff is

$$
R_{t}(\sigma, \tau)=\int_{t}^{\sigma \wedge \tau} f\left(s, Y_{s}, Z_{s}\right) d s+\xi 1_{\{\sigma \wedge \tau=T\}}+L_{\tau} 1_{\{\tau<T, \tau \leq \sigma\}}+U_{\sigma} 1_{\{\sigma<\tau\}},
$$

and a saddle-point $\left(\widehat{\sigma}_{t}, \widehat{\tau}_{t}\right) \in \mathcal{T}_{t} \times \mathcal{T}_{t}$ is given by

$$
\begin{aligned}
& \widehat{\sigma}_{t}=\inf \left\{s \in[t, T) ; Y_{s}=U_{s}\right\} \wedge T, \\
& \widehat{\tau}_{t}=\inf \left\{s \in[t, T) ; Y_{s}=L_{s}\right\} \wedge T .
\end{aligned}
$$




\subsection{Main results}

Our main results in this paper is following:

Theorem 2.1 Under the assumptions 2.1, 2.2 and 2.3, the $R B S D E(\xi, f, L, U)$ has the unique solution $(Y, Z, K)$, which satisfies definition 2.1 (1)-(4).

Proof. Uniqueness. Suppose that the triples $(Y, Z, K)$ and $\left(Y^{\prime}, Z^{\prime}, K^{\prime}\right)$ are two solutions of the $\operatorname{RBSDE}(\xi, f, L)$, i.e. satisfy (1)-(4) of definition 2.1. Set $\Delta Y=Y-Y^{\prime}, \Delta Z=Z-Z^{\prime}$, $\Delta K=\Delta K-\Delta K^{\prime}$, with $\Delta K^{+}=K^{+}-K^{+\prime}, \Delta K^{-}=K^{-}-K^{-\prime}$. Applying Itô's formula to $\Delta Y^{2}$ on the interval $[t, T]$, and taking expectation on both sides, it follows

$$
E\left|\Delta Y_{t}\right|^{2}+E \int_{t}^{T}\left|\Delta Z_{s}\right|^{2} d s \leq 2\left(k^{2}+\mu\right) E \int_{t}^{T} \Delta Y_{s}^{2} d s+\frac{1}{2} E \int_{t}^{T}\left|\Delta Z_{s}\right|^{2} d s
$$

in view of monotonic assumption on $y$, Lipschitz assumption on $z$, and $\int_{t}^{T} \Delta Y_{s} d \Delta K_{s} \leq 0$. We get

$$
E\left|\Delta Y_{t}\right|^{2} \leq 2\left(k^{2}+\mu\right) E \int_{t}^{T} \Delta Y_{s}^{2} d s .
$$

From the Gronwall's inequality, it follows $E\left|\Delta Y_{t}\right|^{2}=E\left|Y_{t}-Y_{t}^{\prime}\right|^{2}=0,0 \leq t \leq T$, i.e. $Y_{t}=Y_{t}^{\prime}$ a.s.; then we have also $E \int_{0}^{T}\left|\Delta Z_{s}\right|^{2} d s=E \int_{0}^{T}\left|Z_{s}-Z_{s}^{\prime}\right|^{2} d s=0$, from which follows $K_{t}=K_{t}^{\prime}$.

Existence. We firstly present the following existence theorem when $f$ does not depend on $z$, which will be proved a little later.

Theorem 2.2 Suppose that $\xi, f$ and $L, U$ satisfy assumption 2.1, 2.2 and 2.3, then for any process $Q \in \mathbf{H}_{d}^{2}(0, T)$, there exists a unique triple of progressively measurable processes $\left\{\left(Y_{t}, Z_{t}, K_{t}\right)_{0 \leq t \leq T}\right\} \in$ $\mathbf{S}^{2}(0, T) \times \mathbf{H}_{d}^{2}(0, T) \times \mathbf{V F}^{2}(0, T)$, with $K=K^{+}-K^{-},\left(K_{t}^{ \pm}\right)_{0 \leq t \leq T} \in \mathbf{A}^{2}(0, T)$, which satisfies 2.1 (1), (3), (4) and

$$
Y_{t}=\xi+\int_{t}^{T} f\left(s, Y_{s}, Q_{s}\right) d s+K_{T}^{+}-K_{t}^{+}-\left(K_{T}^{-}-K_{t}^{-}\right)-\int_{t}^{T} Z_{s} d B_{s}, 0 \leq t \leq T .
$$

Thanks to Theorem 2.2, we can construct a mapping $\Phi$ from $\mathcal{S}$ into itself, where $\mathcal{S}$ is defined as the space of the progressively measurable processes $\left\{\left(Y_{t}, Z_{t}\right) ; 0 \leq t \leq T\right\}$, valued in $\mathbb{R} \times \mathbb{R}^{d}$ which satisfy (1) as follows.

Given $(P, Q) \in \mathcal{S},(Y, Z)=\Phi(P, Q)$ is the unique solution of following RBSDE

$$
Y_{t}=\xi+\int_{t}^{T} f\left(s, Y_{s}, Q_{s}\right) d s+K_{T}-K_{t}-\int_{t}^{T} Z_{s} d B_{s}
$$

i.e., if we define the process

$$
K_{t}=Y_{t}-Y_{0}-\int_{0}^{t} f\left(s, Y_{s}, Q_{s}\right) d s+\int_{0}^{t} Z_{s} d B_{s}, 0 \leq t \leq T,
$$

then the triple $(Y, Z, K)$ satisfies definition 2.1 (1)-(4), with $f(s, y, z)=f\left(s, y, Q_{s}\right)$.

Consider another element of $\mathcal{S}$, and define $\left(Y^{\prime}, Z^{\prime}\right)=\Phi\left(P^{\prime}, Q^{\prime}\right)$; set

$$
\begin{aligned}
& \Delta P=P-P^{\prime}, \Delta Q=Q-Q^{\prime}, \Delta Y=Y-Y^{\prime}, \Delta Z=Z-Z^{\prime}, \\
& \Delta K=K^{+}-K^{-}, \Delta K^{+}=K^{+}-K^{+\prime}, \Delta K^{-}=K^{-}-K^{-\prime} .
\end{aligned}
$$


We apply the Itô's formula to $e^{\gamma t}\left|\Delta Y_{t}\right|^{2}$ on the interval $[t, T]$, for $\gamma>0$,

$$
\begin{gathered}
e^{\gamma t} E\left|\Delta Y_{t}\right|^{2}+E \int_{t}^{T} e^{\gamma s}\left(\gamma\left|\Delta Y_{s}\right|^{2}+\left|\Delta Z_{s}\right|^{2}\right) d s \\
\leq 2\left(k^{2}+\mu\right) E \int_{t}^{T} e^{\gamma s}\left|\Delta Y_{s}\right|^{2} d s+\frac{1}{2} E \int_{t}^{T} e^{\gamma s}\left|\Delta Q_{s}\right|^{2} d s,
\end{gathered}
$$

since $\int_{t}^{T} e^{\gamma s} \Delta Y_{s} d \Delta K_{s}=\int_{t}^{T} e^{\gamma s} \Delta Y_{s} d \Delta K_{s}^{+}-\int_{t}^{T} e^{\gamma s} \Delta Y_{s} d \Delta K_{s}^{-} \leq 0$. Hence, if we choose $\gamma=$ $1+2\left(k^{2}+\mu\right)$, it follows

$$
\begin{aligned}
E \int_{t}^{T} e^{\gamma s}\left(\left|\Delta Y_{s}\right|^{2}+\left|\Delta Z_{s}\right|^{2}\right) d s & \leq \frac{1}{2} E \int_{t}^{T} e^{\gamma s}\left|\Delta Q_{s}\right|^{2} d s \\
& \leq \frac{1}{2} E \int_{t}^{T} e^{\gamma s}\left(\left|\Delta P_{s}\right|^{2}+\left|\Delta Q_{s}\right|^{2}\right) d s
\end{aligned}
$$

Consequently, $\Phi$ is a strict contraction on $\mathcal{S}$ equipped with the norm

$$
\|(Y, Z)\|_{\gamma}=\left[E \int_{0}^{T} e^{\gamma s}\left(\left|Y_{s}\right|^{2}+\left|Z_{s}\right|^{2}\right) d s\right]^{\frac{1}{2}},
$$

and has a fixed point, which is the unique solution of the $\operatorname{RBSDE}(\xi, f, L, U)$.

\subsection{Proof of theorem 2.2}

Now we prove the theorem 2.2 in several steps for the existence of solution. We write $f(s, y)$ for $f\left(s, y, Q_{s}\right)$. First we note that the triple $(Y, Z, K)$ solves the $\operatorname{RBSDE}(\xi, f, L, U), K=K^{+}-K^{-}$, if and only if

$$
\left(\bar{Y}_{t}, \bar{Z}_{t}, \bar{K}_{t}^{+}, \bar{K}_{t}^{-}\right):=\left(e^{\lambda t} Y_{t}, e^{\lambda t} Z_{t}, \int_{0}^{t} e^{\lambda s} d K_{s}^{+}, \int_{0}^{t} e^{\lambda s} d K_{s}^{-}\right)
$$

solves the $\operatorname{RBSDE}(\bar{\xi}, \bar{f}, \bar{L}, \bar{U})$, where

$$
\left(\bar{\xi}, \bar{f}(t, y), \bar{L}_{t}, \bar{U}_{t}\right)=\left(\xi e^{\lambda T}, e^{\lambda t} f\left(t, e^{-\lambda t} y\right)-\lambda y, e^{\lambda t} L_{t}, e^{\lambda t} U_{t}\right) .
$$

If we choose $\lambda=\mu$, then the coefficient $\bar{f}$ satisfies the same assumptions in assumption 2.2 as $f$, but with assumption 2.2-(v) replaced by

$\left(\mathrm{v}^{\prime}\right)\left(y-y^{\prime}\right)\left(f(t, y, z)-f\left(t, y^{\prime}, z\right)\right) \leq 0$.

Since we are in 1-dimensional case, ( $\left.\mathrm{v}^{\prime}\right)$ means that $f$ is decreasing on $y$. From another part the barriers $\bar{L}, \bar{U}$ satisfies:

$\left(i^{\prime}\right)$ :

$$
\begin{aligned}
& E\left[\sup _{0 \leq t \leq T}\left(\bar{L}_{t}\right)^{+}\right]<\infty, E\left[\varphi^{2}\left(\sup _{0 \leq t \leq T}\left(\bar{L}_{t}\right)^{+}\right)\right]=E\left[\varphi^{2}\left(\sup _{0 \leq t \leq T}\left(e^{\mu t}\left(L_{t}\right)^{+}\right)\right)\right]<\infty, \\
& E\left[\sup _{0 \leq t \leq T}\left(\bar{U}_{t}\right)^{-}\right]<\infty, E\left[\varphi^{2}\left(\sup _{0 \leq t \leq T}\left(\bar{U}_{t}\right)^{-}\right)\right]=E\left[\varphi^{2}\left(\sup _{0 \leq t \leq T}\left(e^{\mu t}\left(U_{t}\right)^{-}\right)\right)\right]<\infty .
\end{aligned}
$$

In the following, we shall work with assumption $2.2^{\prime}$ which is assumption 2.2 with (v) replaced by (v') and assumption 2.3' which is assumption 2.2 with (i') instead of (i).

Proof of Theorem 2.2; First, let us recall the assumptions on the coefficient $f$ : 
Assumption 2.4. For $y \in \mathbb{R}, s \in[0, T]$,

(i) $\quad|f(s, y)| \leq|f(s, 0,0)|+k\left|Q_{s}\right|+\varphi(|y|)$;

(ii) $E \int_{0}^{T}|f(t, 0)|^{2} d t<\infty$;

(iii) $\left(y-y^{\prime}\right)\left(f(s, y)-f\left(s, y^{\prime}\right)\right) \leq 0$;

(iv) $y \rightarrow f(s, y)$ is continuous, a.s..

We point out that we always denote by $c>0$ a constant whose value can be changed line by line. The proof will be done by five steps as following.

- Using a penalization method we prove the existence under the assumption

$$
|\xi|+\sup _{0 \leq t \leq T}|f(t, 0)|+\sup _{0 \leq t \leq T} L_{t}^{+}+\sup _{0 \leq t \leq T} U_{t}^{-} \leq c .
$$

- Approximating the lower barrier $L$, we prove the existence under the assumption that $L$ satisfies assumption 2.3'-(i) and the bounded assumption on $\xi, f(t, 0)$ and $\sup _{0 \leq t \leq T} U_{t}^{-}$.

- Like above step, we approximate the upper barrier $U$ to prove the existence under assumption 2.3 ' and $\xi$ and $f(t, 0)$ satisfy

$$
|\xi|^{2}+\sup _{0 \leq t \leq T}|f(t, 0)|^{2} \leq c
$$

- By approximation, we prove the existence of the solution under the assumption $\xi \geq c$, $\inf _{0 \leq t \leq T} f(t, 0) \geq c$.

- Finally, we prove the existence of the solution under the assumption $\xi \in \mathbf{L}^{2}\left(\mathcal{F}_{T}\right), f(t, 0) \in$ $\mathbf{H}^{2}(0, T)$, by approximation.

In each step, we use monotonic property of approximation solutions to get the convergence.

Step 1. Consider the penalization equations with respect to the two barriers $L, U$, for $m, n \in \mathbf{N}$,

$$
Y_{t}^{m, n}=\xi+\int_{t}^{T} f\left(s, Y_{s}^{m, n}\right) d s+m \int_{t}^{T}\left(Y_{s}^{m, n}-L_{s}\right)^{-} d s-n \int_{t}^{T}\left(U_{s}-Y_{s}^{m, n}\right)^{-} d s-\int_{t}^{T} Z_{s}^{m, n} d B_{s} .
$$

Set $f_{m, n}(s, y)=f(s, y)+m\left(y-L_{s}\right)^{-}-n\left(U_{s}-y\right)^{-}$, obviously, $f_{m, n}$ satisfies the condition of Proposition 2.4 in [13]. So by the Proposition 2.4 in [13], there exists $\left(Y_{t}^{m, n}, Z_{t}^{m, n}\right)_{0 \leq t \leq T}$, which is the solution of (5). Denote $K_{t}^{m, n,+}=m \int_{0}^{t}\left(Y_{s}^{m, n}-L_{s}\right)^{-} d s, K_{t}^{m, n,-}=n \int_{0}^{t}\left(U_{s}-Y_{s}^{m, n}\right)^{-} d s$.

Now let us do the uniformly a priori estimation of $\left(Y^{m, n}, Z^{m, n}, K^{m, n,+}, K^{m, n,-}\right)$.

Lemma 2.1 There exists a constant $C_{0}$ independent of $n$, such that

$$
E\left[\sup _{0 \leq t \leq T}\left|Y_{t}^{m, n}\right|^{2}+\int_{0}^{T}\left|Z_{s}^{m, n}\right|^{2} d s+\left(K_{T}^{m, n,+}\right)^{2}+\left(K_{T}^{m, n,-}\right)^{2}\right] \leq C_{0} .
$$

Proof. Consider the $\operatorname{RBSDE}(\xi, f, L)$ with one lower barrier $L$; due to theorem 2.3 in [11, it admits a unique solution $\left(\bar{Y}_{t}, \bar{Z}_{t}, \bar{K}_{t}\right)_{0 \leq t \leq T} \in \mathbf{S}^{2}(0, T) \times \mathbf{H}_{d}^{2}(0, T) \times \mathbf{A}^{2}(0, T)$, which satisfies

$$
\bar{Y}_{t}=\xi+\int_{t}^{T} f\left(s, \bar{Y}_{s}\right) d s+\bar{K}_{T}-\bar{K}_{t}-\int_{t}^{T} \bar{Z}_{s} d B_{s}
$$


$\bar{Y}_{t} \geq L_{t}, 0 \leq t \leq T, \int_{0}^{T}\left(\bar{Y}_{s}-L_{s}\right) d \bar{K}_{s}=0$. In order to compare (6) and (5) , we consider the penalization equation associated with the RBSDE ([6]), for $m \in \mathbf{N}$,

$$
\bar{Y}_{t}^{m}=\xi+\int_{t}^{T} f\left(s, \bar{Y}_{s}^{m}\right) d s+m \int_{t}^{T}\left(L_{s}-\bar{Y}_{s}^{m}\right)^{+} d s-\int_{t}^{T} \bar{Z}_{s}^{m} d B_{s}
$$

Comparing (15) and (7), we get $Y_{t}^{m, n} \leq \bar{Y}_{t}^{m}, \forall t \in[0, T], n \in \mathbf{N}$. Thank to the convergence result of step1 and step 2 in the proof of theorem 2.3 in [11, i.e. $\bar{Y}^{m} \rightarrow \bar{Y}$ in $\mathbf{S}^{2}(0, T)$. So we get for any $m, n \in \mathbf{N}, t \in[0, T], Y_{t}^{m, n} \leq \bar{Y}_{t}$.

Similarly, we consider the $\operatorname{RBSDE}(\xi, f, U)$ with one upper barrier $U$. There exists $\left(\underline{Y}_{t}, \underline{Z}_{t}, \underline{K}_{t}\right)_{0 \leq t \leq T} \in$ $\mathbf{S}^{2}(0, T) \times \mathbf{H}_{d}^{2}(0, T) \times \mathbf{A}^{2}(0, T)$, which satisfies

$$
\underline{Y}_{t}=\xi+\int_{t}^{T} f\left(s, \underline{Y}_{s}\right) d s-\left(\underline{K}_{T}-\underline{K}_{t}\right)-\int_{t}^{T} \underline{Z}_{s} d B_{s}
$$

$\underline{Y}_{t} \leq U_{t}, 0 \leq t \leq T, \int_{0}^{T}\left(\underline{Y}_{s}-U_{s}\right) d \underline{K}_{s}=0$. By the penalization equation associated with (요 $)$ and the comparison theorem, we deduce that $Y_{t}^{m, n} \geq \underline{Y}_{t}$, for any $m, n \in \mathbf{N}, t \in[0, T]$. Then we get, with the results of the step 1 in the proof of theorem 2.3 [11],

$$
\sup _{0 \leq t \leq T}\left|Y_{t}^{m, n}\right| \leq \max \left\{\sup _{0 \leq t \leq T}\left|\bar{Y}_{t}\right|, \sup _{0 \leq t \leq T}\left|\underline{Y}_{t}\right|\right\} \leq C .
$$

In the following, notice that assumption 2.4-(iii) implies that $f$ is decreasing on $y$, for $s \in[0, T]$, so $f\left(s, \underline{Y}_{s}\right) \geq f\left(s, Y_{s}^{m, n}\right) \geq f\left(s, \bar{Y}_{s}\right)$, with the square-integrable results of (마) and (으), it follows

$$
\left|f\left(s, Y_{s}^{m, n}\right)\right| \leq \max \left\{\left|f\left(s, \bar{Y}_{s}\right)\right|,\left|f\left(s, \underline{Y}_{s}\right)\right|\right\} \leq C .
$$

To get the estimation of $\left(K^{m, n,+}, K^{m, n,-}, Z^{m, n}\right)$, we apply Itô's formula to $\left(Y^{m, n}\right)^{2}$, then

$$
\begin{aligned}
& E\left(Y_{t}^{m, n}\right)^{2}+E \int_{t}^{T}\left|Z_{s}^{m, n}\right|^{2} d s \\
\leq & E\left[\xi^{2}\right]+E \int_{t}^{T}\left|Y_{s}^{m, n}\right|^{2} d s+E \int_{t}^{T}|f(s, 0)|^{2} d s+\frac{1}{\alpha} E\left[\sup _{0 \leq t \leq T}\left(L_{t}^{+}\right)^{2}\right]+\frac{1}{\alpha} E\left[\sup _{0 \leq t \leq T}\left(U_{t}^{-}\right)^{2}\right] \\
& +\alpha E\left[m \int_{t}^{T}\left(L_{s}-Y_{s}^{m, n}\right)^{+} d s\right]^{2}+\alpha E\left[n \int_{t}^{T}\left(U_{s}-Y_{s}^{m, n}\right)^{-} d s\right]^{2},
\end{aligned}
$$

for some $\alpha>0$, in view of

$\int_{t}^{T} Y_{s}^{m, n}\left(L_{s}-Y_{s}^{m, n}\right)^{+} d s=\int_{t}^{T} L_{s}\left(L_{s}-Y_{s}^{m, n}\right)^{+} d s-\int_{t}^{T}\left(\left(L_{s}-Y_{s}^{m, n}\right)^{+}\right)^{2} d s \leq \int_{t}^{T} L_{s}\left(L_{s}-Y_{s}^{m, n}\right)^{+} d s$, and $\int_{t}^{T} Y_{s}^{m, n}\left(U_{s}-Y_{s}^{m, n}\right)^{-} d s \leq \int_{t}^{T} U_{s}\left(U_{s}-Y_{s}^{m, n}\right)^{-} d s$. So

$$
E \int_{t}^{T}\left|Z_{s}^{m, n}\right|^{2} d s \leq C+\alpha\left(E\left[m \int_{t}^{T}\left(L_{s}-Y_{s}^{m, n}\right)^{+} d s\right]^{2}+E\left[n \int_{t}^{T}\left(U_{s}-Y_{s}^{m, n}\right)^{-} d s\right)^{2}\right] .
$$

We need to prove that there exists a constant $C$ independent of $m, n$ such that for any $0 \leq t \leq T$

$$
E\left[m \int_{t}^{T}\left(L_{s}-Y_{s}^{m, n}\right)^{+} d s\right]^{2}+E\left[n \int_{t}^{T}\left(U_{s}-Y_{s}^{m, n}\right)^{-} d s\right]^{2} \leq C+8 E \int_{t}^{T}\left|Z_{s}^{m, n}\right|^{2} d s .
$$


In fact, let us consider the stopping time

$$
\begin{aligned}
& \tau_{1}=\inf \left\{r \geq t \mid Y_{r}^{m, n} \geq U_{r}\right\} \wedge T, \sigma_{1}=\inf \left\{r \geq \tau_{1} \mid Y_{r}^{m, n}=L_{r}\right\} \wedge T, \\
& \tau_{2}=\inf \left\{r \geq \sigma_{1} \mid Y_{r}^{m, n}=U_{r}\right\} \wedge T,
\end{aligned}
$$

and so on. Since $L<U$ on $[0, T)$, and $L$ and $U$ are continuous, then when $k \rightarrow \infty$, we have $\tau_{k} \nearrow T$, $\sigma_{k} \nearrow T$. Obviously $Y^{m, n} \geq L$ on the interval $\left[\tau_{k}, \sigma_{k}\right]$, so we get

$$
Y_{\tau_{k}}^{m, n}=Y_{\sigma_{k}}^{m, n}+\int_{\tau_{k}}^{\sigma_{k}} f\left(s, Y_{s}^{m, n}\right) d s-n \int_{\tau_{k}}^{\sigma_{k}}\left(Y_{s}^{m, n}-U_{s}\right)^{+} d s-\int_{\tau_{k}}^{\sigma_{k}} Z_{s}^{m, n} d B_{s} .
$$

On the other hand

$$
\begin{aligned}
& Y_{\tau_{k}}^{m, n} \geq J_{\tau_{k}}, \text { on }\left\{\tau_{k}<T\right\}, Y_{\tau_{k}}^{m, n}=J_{\tau_{k}}=\xi, \text { on }\left\{\tau_{k}=T\right\}, \\
& Y_{\sigma_{k}}^{m, n} \leq J_{\sigma_{k}}, \text { on }\left\{\sigma_{k}<T\right\}, Y_{\sigma_{k}}^{m, n}=J_{\sigma_{k}}=\xi, \text { on }\left\{\sigma_{k}=T\right\}
\end{aligned}
$$

and these inequalities imply that for all $k$, the following holds

$$
\begin{aligned}
n \int_{\tau_{k}}^{\sigma_{k}}\left(Y_{s}^{m, n}-U_{s}\right)^{+} d s & \leq J_{\sigma_{k}}-J_{\tau_{k}}+\int_{\tau_{k}}^{\sigma_{k}} f\left(s, Y_{s}^{m, n}\right) d s-\int_{\tau_{k}}^{\sigma_{k}} Z_{s}^{m, n} d B_{s} \\
& \leq \int_{\tau_{k}}^{\sigma_{k}}\left(\phi_{s}-Z_{s}^{m, n}\right) d B_{s}+V_{\sigma_{k}}^{-}-V_{\tau_{k}}^{-}+\int_{\tau_{k}}^{\sigma_{k}}\left|f\left(s, Y_{s}^{m, n}\right)\right| d s .
\end{aligned}
$$

Notice that on the interval $\left[\sigma_{k}, \tau_{k+1}\right], Y_{s}^{m, n} \leq U_{s}$; we obtain by summing in $k$

$$
n \int_{t}^{T}\left(Y_{s}^{m, n}-U_{s}\right)^{+} d s \leq \int_{t}^{T}\left(\left(\phi_{s}-Z_{s}^{m, n}\right)\left(\sum_{k} 1_{\left[\tau_{k}, \sigma_{k}\right)}(s)\right) d B_{s}+V_{T}^{-}+\int_{t}^{T}\left|f\left(s, Y_{s}^{m, n}\right)\right| d s .\right.
$$

By squaring and taking the expectation, with (10), we get

$$
\begin{aligned}
& E\left[n \int_{t}^{T}\left(Y_{s}^{m, n}-U_{s}\right)^{+} d s\right]^{2} \\
\leq & 4 E \int_{t}^{T}\left|\phi_{s}\right|^{2} d s+4 E \int_{t}^{T}\left|Z_{s}^{m, n}\right|^{2} d s+2 E\left[\left(V_{T}^{-}\right)^{2}\right]+2 E\left(\int_{t}^{T}\left|f\left(s, Y_{s}^{m, n}\right)\right| d s\right)^{2} \\
\leq & C+4 E \int_{t}^{T}\left|Z_{s}^{m, n}\right|^{2} d s
\end{aligned}
$$

in the same way, we obtain

$$
E\left[m \int_{t}^{T}\left(L_{s}-Y_{s}^{m, n}\right)^{+} d s\right]^{2} \leq C+4 E \int_{t}^{T}\left|Z_{s}^{m, n}\right|^{2} d s .
$$

By (12) and (13), and (11), with $\alpha=\frac{1}{16}$, it follows

$$
E \int_{t}^{T}\left|Z_{s}^{m, n}\right|^{2} d s \leq C
$$

then

$$
E\left[\left(K_{T}^{m, n,+}\right)^{2}+\left(K_{T}^{m, n,-}\right)^{2}\right] \leq C
$$


Let $m \rightarrow \infty$, due to the convergence results in step 1 of the proof in [1], $Y^{m, n} \rightarrow Y^{n}$ in $\mathbf{S}^{2}(0, T), K^{m, n,+} \rightarrow K^{n,+}$ in $\mathbf{A}^{2}(0, T)$, and $Z^{m, n} \rightarrow Z^{n}$ in $\mathbf{H}_{d}^{2}(0, T)$, where $\left(Y^{n}, Z^{n}, K^{n,+}\right)$ is the solution of the one lower barrier $\operatorname{RBSDE}\left(\xi, f_{n}, L\right)$, with $f_{n}(s, y)=f(s, y)-n\left(y-U_{s}\right)^{+}$. So

$$
Y_{t}^{n}=\xi+\int_{t}^{T} f\left(s, Y_{s}^{n}\right) d s+K_{T}^{n,+}-K_{t}^{n,+}-n \int_{t}^{T}\left(Y_{s}^{n}-U_{s}\right)^{+} d s-\int_{t}^{T} Z_{s}^{n} d B_{s},
$$

$Y_{t}^{n} \geq L_{t}, 0 \leq t \leq T, \int_{0}^{T}\left(Y_{s}^{n}-L_{s}\right) d K_{s}^{n}=0$. Thank to the uniform estimations, which we got as above, we know that there exists a constant $C$ independent of $n$ and $t$, s.t.

$$
\sup _{0 \leq t \leq T}\left(Y_{t}^{n}\right)^{2}+f\left(t, Y_{t}^{n}\right) \leq C
$$

and

$$
E \int_{0}^{T}\left|Z_{s}^{n}\right|^{2} d s+E\left[\left(K_{T}^{n,+}\right)^{2}\right]+E\left[\left(K_{T}^{n,-}\right)^{2}\right] \leq C
$$

where $K_{t}^{n,-}=n \int_{0}^{t}\left(Y_{s}^{n}-U_{s}\right)^{+} d s$. Then by the comparison theorem 4.3 in [1], we deduce that $Y_{t}^{n} \searrow Y_{t}$, for $t \in[0, T]$, as $n \rightarrow \infty$, and by the dominated convergence theorem

$$
E \int_{0}^{T}\left(Y_{s}^{n}-Y_{s}\right)^{2} d s \rightarrow 0, \text { as } n \rightarrow \infty
$$

Then we want to prove the convergence of $\left(Z^{n}\right)$ in $\mathbf{H}_{d}^{2}(0, T)$. For this, we need the following lemma, which is analogue as Lemma 4 in [12. With (10), (9) and (3), we can easily get it, so we omit the proof.

\section{Lemma 2.2}

$$
\lim _{n \rightarrow \infty} E\left(\sup _{0 \leq t \leq T}\left(\left(Y_{t}^{n}-U_{t}\right)^{+}\right)^{2}=0 .\right.
$$

For $n, p \in \mathbf{N}$, applying Itô's formula to $\left|Y^{n}-Y^{p}\right|^{2}$, and taking the expectation, then

$$
\begin{aligned}
& E\left(Y_{t}^{n}-Y_{t}^{p}\right)^{2}+E \int_{t}^{T}\left|Z_{s}^{n}-Z_{s}^{p}\right|^{2} d s \\
\leq & 2 E \int_{t}^{T}\left(Y_{s}^{n}-U_{s}\right)^{+} d K_{s}^{p,-}+2 E \int_{t}^{T}\left(Y_{s}^{p}-U_{s}\right)^{+} d K_{s}^{n,-} \\
\leq & 2\left(E\left[\left(\sup _{0 \leq t \leq T}\left(Y_{s}^{n}-U_{s}\right)^{+}\right)^{2}\right]\right)^{\frac{1}{2}}\left(E\left(K_{T}^{p,-}\right)^{2}\right)^{\frac{1}{2}}+2\left(E\left[\left(\sup _{0 \leq t \leq T}\left(Y_{s}^{p}-U_{s}\right)^{+}\right)^{2}\right]\right)^{\frac{1}{2}}\left(E\left(K_{T}^{n,-}\right)^{2}\right)^{\frac{1}{2}},
\end{aligned}
$$

since $\int_{t}^{T}\left(Y_{s}^{n}-Y_{s}^{p}\right) d\left(K_{s}^{n,+}-K_{s}^{p,+}\right) \leq 0$. So by (19) and (17), as $n, p \rightarrow \infty, E \int_{t}^{T}\left|Z_{s}^{n}-Z_{s}^{p}\right|^{2} d s \rightarrow 0$, which implies $\left\{Z^{n}\right\}$ is a Cauchy sequence in $\mathbf{H}_{d}^{2}(0, T)$. So there exists a process $Z \in \mathbf{H}_{d}^{2}(0, T)$, s.t., as $n \rightarrow \infty$,

$$
E \int_{t}^{T}\left|Z_{s}^{n}-Z_{s}\right|^{2} d s \rightarrow 0 .
$$

Moreover by Itô's formula, we have

$$
\begin{aligned}
E\left[\sup _{0 \leq t \leq T}\left|Y_{t}^{n}-Y_{t}^{p}\right|^{2}\right] \leq & 2 E \int_{t}^{T}\left(Y_{s}^{n}-U_{s}\right)^{+} d K_{s}^{p,-}+2 E \int_{t}^{T}\left(Y_{s}^{p}-U_{s}\right)^{+} d K_{s}^{n,-} \\
& +2 E\left[\sup _{0 \leq t \leq T} \int_{t}^{T}\left|Z_{s}^{n}-Z_{s}^{p}\right|\left|Y_{s}^{n}-Y_{s}^{p}\right| d B_{s}\right] .
\end{aligned}
$$


By Burkholder-Davis-Gundy inequality and (19), we get, as $n, p \rightarrow \infty$

$$
E\left[\sup _{0 \leq t \leq T}\left|Y_{t}^{n}-Y_{t}^{p}\right|^{2}\right] \rightarrow 0
$$

i.e. $Y^{n} \searrow Y$, in $\mathbf{S}^{2}(0, T)$.

By the convergence of $Y_{t}^{n}$, i.e. $Y_{t}^{n} \searrow Y_{t}, 0 \leq t \leq T$, and the fact that $f(s, y)$ is continuous and decreasing in $y$, we get $f\left(s, Y_{s}^{n}\right) \nearrow f\left(s, Y_{s}\right), 0 \leq s \leq T$. Moreover $\left|f\left(s, Y_{s}^{n}\right)\right| \leq C$. Using the monotonic convergence theorem, we deduce that

$$
E \int_{0}^{T}\left[f\left(t, Y_{t}^{n}\right)-f\left(t, Y_{t}\right)\right]^{2} d t \rightarrow 0
$$

i.e. the sequence $\left\{f\left(\cdot, Y^{n}\right)\right\}$ is also a Cauchy sequence in $\mathbf{H}^{2}(0, T)$.

Now we consider the convergence of the increasing processes $\left(K^{n,+}\right)$ and $\left(K^{n,-}\right)$. By the comparison theorem 4.3 in [11], we get $K_{t}^{n,+} \geq K_{t}^{p,+}, K_{t}^{n,+}-K_{s}^{n,+} \geq K_{t}^{p,+}-K_{s}^{p,+}$, for $0 \leq s \leq t \leq T$. So for $0 \leq t \leq T, K_{t}^{n,+} \nearrow K_{t}^{+}$, with $E\left[\left(K_{t}^{n,+}\right)^{2}\right] \leq C$, we get that $E\left[\left(K_{t}^{+}\right)^{2}\right] \leq C$. Furthermore, $K_{T}^{n,+}-K_{T}^{p,+} \geq K_{t}^{n,+}-K_{t}^{p,+}$, which follows

$$
E\left[\sup _{0 \leq t \leq T}\left(K_{t}^{n,+}-K_{t}^{p,+}\right)^{2}\right] \leq E\left[\left(K_{T}^{n,+}-K_{T}^{p,+}\right)^{2}\right] \rightarrow 0,
$$

so $K^{n,+} \rightarrow K^{+}$in $\mathbf{A}^{2}(0, T)$. On the other hand, since $\left(Y^{n}, Z^{n}, K^{n+}, K^{n-}\right)$ satisfies

$$
Y_{t}^{n}=\xi+\int_{t}^{T} f\left(s, Y_{s}^{n}\right) d s+K_{T}^{n,+}-K_{t}^{n,+}-\left(K_{T}^{n,-}-K_{t}^{n,-}\right)-\int_{t}^{T} Z_{s}^{n} d B_{s}
$$

and we can rewrite it in the following form

$$
K_{t}^{n,-}=Y_{t}^{n}-Y_{0}^{n}+\int_{0}^{t} f\left(s, Y_{s}^{n}\right) d s+K_{t}^{n,+}-\int_{0}^{t} Z_{s}^{n} d B_{s} .
$$

Without losing the generality, for $p<n$, with BDG inequality, we get

$$
\begin{aligned}
& E\left[\sup _{0 \leq t \leq T}\left(K_{t}^{n,-}-K_{t}^{p,-}\right)^{2}\right] \\
\leq & 5 E\left[\sup _{0 \leq t \leq T}\left(Y_{t}^{n}-Y_{t}^{p}\right)^{2}\right]+5\left(Y_{0}^{n}-Y_{0}^{p}\right)^{2}+5 T E\left(\int_{0}^{T}\left(f\left(s, Y_{s}^{n}\right)-f\left(s, Y_{s}^{p}\right)\right)^{2} d s\right) \\
& +5 E\left[\left(K_{T}^{n,+}-K_{T}^{p,+}\right)^{2}\right]+C E \int_{0}^{t}\left(Z_{s}^{n}-Z_{s}^{p}\right)^{2} d s \\
\rightarrow & 0,
\end{aligned}
$$

i.e. there exists a process $K^{-} \in \mathbf{A}^{2}(0, T)$, s.t. $K^{n,-} \rightarrow K^{-}$in $\mathbf{A}^{2}(0, T)$, and the limit $\left(Y, Z, K^{+}, K^{-}\right)$ satisfies

$$
Y_{t}=\xi+\int_{t}^{T} f\left(s, Y_{s}\right) d s+K_{T}^{+}-K_{t}^{+}-\left(K_{T}^{-}-K_{t}^{-}\right)-\int_{t}^{T} Z_{s} d B_{s} .
$$

Since for $n \in \mathbf{N}, Y_{t}^{n} \geq L_{t}, 0 \leq t \leq T$, so $Y_{t} \geq L_{t}$. The last is to check (4) of definition 2.1. Since $\left(Y^{n}, K^{n,+}, K^{n,-}\right)$ tends to $\left(Y, K^{+}, K^{-}\right)$uniformly in $t$ in probability, then the measure $d K^{n,+}$ converges to $d K^{+}$weakly in probability, so

$$
\int_{0}^{T}\left(Y_{t}^{n}-L_{t}\right) d K_{t}^{n,+} \rightarrow \int_{0}^{T}\left(Y_{t}-L_{t}\right) d K_{t}^{+}
$$


in probability as $n \rightarrow \infty$. Obviously $\int_{0}^{T}\left(Y_{t}-L_{t}\right) d K_{t}^{+} \geq 0$, On the other hand, for each $n \in \mathbf{N}$, $\int_{0}^{T}\left(Y_{t}^{n}-L_{t}\right) d K_{t}^{n,+}=0$. Hence

$$
\int_{0}^{T}\left(Y_{t}-L_{t}\right) d K_{t}^{+}=0, \text { a.s. }
$$

Similarly, we have $\int_{0}^{T}\left(Y_{t}-U_{t}\right) d K_{t}^{-}=0$. Consequently the triple $\left(Y, Z, K^{+}, K^{-}\right)$is solution of the $\operatorname{RBSDE}(\xi, f, L, U)$, under the assumptions (3).

Step 2. In this step, we consider the case of a barrier $L$ which satisfies the assumption 2.3'-(i):

$$
E\left[\varphi^{2}\left(\sup _{0 \leq t \leq T}\left(L_{t}\right)^{+}\right)\right]<\infty
$$

and $L^{+} \in \mathbf{S}^{2}(0, T)$, but we still assume that for some $C>0$,

$$
|\xi|+\sup _{0 \leq t \leq T}|f(t, 0)|+\sup _{0 \leq t \leq T}\left(U_{t}\right)^{-} \leq C .
$$

For $n \in \mathbf{N}$, set $L^{n}=L \wedge n$, then $\sup _{0 \leq t \leq T}\left(L_{t}^{n}\right)^{+} \leq n$ and $L_{t}^{n} \leq L_{t}$; so assumption 2.3'(ii), (iii) are satisfied and by the step 1, we know that there exists a triple $\left(Y^{n}, Z^{n}, K^{n}\right)$, with $K^{n}=K^{n,+}-K^{n,-}$, which satisfies

$$
Y_{t}^{n}=\xi+\int_{t}^{T} f\left(s, Y_{s}^{n}\right) d s+K_{T}^{n,+}-K_{t}^{n,+}-\left(K_{T}^{n,-}-K_{t}^{n,-}\right)-\int_{t}^{T} Z_{s}^{n} d B_{s},
$$

$L_{t}^{n} \leq Y_{t}^{n} \leq U_{t}, 0 \leq t \leq T$, and $\int_{0}^{T}\left(Y_{t}^{n}-L_{t}^{n}\right) d K_{t}^{n,+}=\int_{0}^{T}\left(Y_{t}^{n}-U_{t}\right) d K_{t}^{n,-}=0$.

Consider the solution $(\bar{Y}, \bar{Z}, \bar{K})$ of one lower barrier $\operatorname{RBSDE}(\xi, f, L)$ and the solution $(\underline{Y}, \underline{Z}, \underline{K})$ of the super barrier $\operatorname{RBSDE}(\xi, f, U)$, in fact these two equations can be considered as the following two barriers $\operatorname{RBSDE}(\xi, f, L, \bar{U})$ and $\operatorname{RBSDE}(\xi, f, \underline{L}, U)$, where $\underline{L}=-\infty, \bar{U}=+\infty$. By the comparison theorem 3.3 , it follows that $\underline{Y}_{t} \leq Y_{t}^{n} \leq \bar{Y}_{t}, 0 \leq t \leq T$. So

$$
E\left[\sup _{0 \leq t \leq T}\left|Y_{t}^{n}\right|^{2}\right] \leq \max \left\{E \left[\sup _{0 \leq t \leq T}\left|\bar{Y}_{t}\right|^{2}, E\left[\sup _{0 \leq t \leq T}\left|\underline{Y}_{t}\right|^{2}\right\} \leq C .\right.\right.
$$

Since $L_{t}^{n} \leq L_{t}^{n+1}, 0 \leq t \leq T$, thanks to the comparison theorem 3.3, $Y_{t}^{n} \nearrow Y_{t}, 0 \leq t \leq T$. From the above estimate and Fatou's lemma, we get

$$
E\left[\sup _{0 \leq t \leq T}\left(Y_{t}\right)^{2}\right] \leq C
$$

And

$$
E \int_{0}^{T}\left|Y_{t}^{n}-Y_{t}\right|^{2} d t \rightarrow 0, \text { as } n \rightarrow \infty,
$$

follows from the dominated convergence theorem.

Notice that $f$ is decreasing on $y$, then $f\left(t, \bar{Y}_{t}\right) \leq f\left(t, Y_{t}^{n}\right) \leq f\left(t, \underline{Y}_{t}\right), 0 \leq t \leq T$, and with the integral property of $\underline{Y}$ and $\bar{Y}$, we have

$$
E\left[\left(\int_{0}^{t} f\left(s, Y_{s}^{n}\right) d s\right)^{2}\right] \leq \max \left\{E\left[\left(\int_{0}^{t} f\left(s, \bar{Y}_{s}\right) d s\right)^{2}\right], E\left[\left(\int_{0}^{t} f\left(s, \underline{Y}_{s}\right) d s\right)^{2}\right]\right\} \leq C .
$$


In order to prove the convergence of $\left(Z^{n}, K^{n}\right)$, we first need a-priori estimations. We apply the Itô formula to $\left|Y_{t}^{n}\right|^{2}$ on the interval $[t, T]$,

$$
\begin{aligned}
& E\left|Y_{t}^{n}\right|^{2}+E \int_{t}^{T}\left|Z_{s}^{n}\right|^{2} d s \\
\leq & E|\xi|^{2}+E \int_{t}^{T}\left|Y_{s}^{n}\right|^{2} d s+E \int_{t}^{T}|f(s, 0)|^{2} d s+(\alpha+\beta) E\left[\sup _{0 \leq t \leq T}\left|Y_{t}^{n}\right|^{2}\right] \\
+ & \frac{1}{\alpha} E\left[\left(K_{T}^{n,+}-K_{t}^{n,+}\right)^{2}\right]+\frac{1}{\beta} E\left[\left(K_{T}^{n,-}-K_{t}^{n,-}\right)^{2}\right],
\end{aligned}
$$

where $K^{n}=K^{n,+}-K^{n,-}$. We first use the comparison theorem to estimate $K^{n,-}$. Consider the linear $\operatorname{RBSDE}\left(\xi, f\left(s, L_{s}{ }^{-}\right), L, U\right)$, by existence results of [4], we know there exists $\left(\widetilde{Y}, \widetilde{Z}, \widetilde{K}^{+}, \widetilde{K}^{-}\right) \in$ $\mathbf{S}^{2}(0, T) \times \mathbf{H}_{d}^{2}(0, T) \times \mathbf{A}^{2}(0, T) \times \mathbf{A}^{2}(0, T)$ satisfying

$$
\begin{aligned}
& \widetilde{Y}_{t}=\xi+\int_{t}^{T} f\left(s,\left(L_{s}\right)^{-}\right) d s+\widetilde{K}_{T}^{+}-\widetilde{K}_{t}^{+}-\left(\widetilde{K}_{T}^{-}-\widetilde{K}_{t}^{-}\right)-\int_{t}^{T} \widetilde{Z}_{s} d B_{s} \\
& L_{t} \leq \widetilde{Y}_{t} \leq U_{t}, \int_{0}^{T}\left(\widetilde{Y}_{t}-L_{t}\right) d \widetilde{K}_{t}^{+}=\int_{0}^{T}\left(\widetilde{Y}_{t}-U_{t}\right) d \widetilde{K}_{t}^{-}=0 .
\end{aligned}
$$

Then we have the following lemma, which will be proved in Appendix.

Lemma 2.3 For $0 \leq s \leq t \leq T, K_{t}^{n,-}-K_{s}^{n,-} \leq \widetilde{K}_{t}^{-}-\widetilde{K}_{s}^{-}$, and $K_{T}^{n,-} \leq \widetilde{K}_{T}^{-}$.

Now we have

$$
E\left[\left(K_{T}^{n,-}\right)^{2}\right] \leq E\left[\left(\widetilde{K}_{T}^{-}\right)^{2}\right] \leq C .
$$

We rewrite the $\operatorname{RBSDE}\left(\xi, f, L^{n}, U\right)(22)$,

$$
K_{T}^{n,+}-K_{t}^{n,+}=Y_{t}^{n}-\xi-\int_{t}^{T} f\left(s, Y_{s}^{n}\right) d s+\left(K_{T}^{n,-}-K_{t}^{n,-}\right)+\int_{t}^{T} Z_{s}^{n} d B_{s},
$$

hence

$$
\begin{aligned}
E\left(K_{T}^{n,+}-K_{t}^{n,+}\right)^{2} \leq & 5 E\left|Y_{t}^{n}\right|^{2}+5 E|\xi|^{2}+5 E\left(\int_{t}^{T} f\left(s, Y_{s}^{n}\right) d s\right)^{2} \\
& +5 E\left[\left(K_{T}^{n,-}-K_{t}^{n,-}\right)^{2}\right]+5 E \int_{t}^{T}\left|Z_{s}^{n}\right|^{2} d s \\
\leq & C+5 E \int_{t}^{T}\left|Z_{s}^{n}\right|^{2} d s .
\end{aligned}
$$

Then we substitute (28) into (27), set $\alpha=10, \beta=1$, and with (21) and (23), it follows

$$
E\left(K_{T}^{n,+}\right)^{2}+E \int_{0}^{T}\left|Z_{s}^{n}\right|^{2} d s \leq C .
$$

Now for $n, p \in \mathbf{N}, n \geq p$, then $L_{t}^{n} \geq L_{t}^{p}, 0 \leq t \leq T$. We apply the Itô's formula to $\left(\left|Y_{t}^{n}-Y_{t}^{p}\right|^{2}\right)$ on the interval $[t, T]$, and take expectation

$$
\begin{aligned}
E\left[\left|Y_{t}^{n}-Y_{t}^{p}\right|^{2}\right]+E \int_{t}^{T}\left|Z_{s}^{n}-Z_{s}^{p}\right|^{2} d s & \leq 2 E \int_{t}^{T}\left(L_{s}^{n}-L_{s}^{p}\right) d K_{s}^{n,+}-2 E \int_{t}^{T}\left(L_{s}^{n}-L_{s}^{p}\right) d K_{s}^{p,+} \\
& \leq 2 E \int_{t}^{T}\left(L_{s}^{n}-L_{s}^{p}\right) d K_{s}^{n,+}
\end{aligned}
$$


in view of $\int_{t}^{T}\left(Y_{s}^{n}-Y_{s}^{p}\right) d\left(K_{s}^{n,-}-K_{s}^{p,-}\right) \geq 0$. Since $L_{t}-L_{t}^{n} \downarrow 0$, for each $t \in[0, T]$, and $L_{t}-L_{t}^{n}$ is continuous, by the Dini's theorem, the convergence holds uniformly on the interval $[0, T]$, i.e.

$$
E\left[\sup _{0 \leq t \leq T}\left(L_{t}-L_{t}^{n}\right)^{2}\right] \rightarrow 0, \text { as } n \rightarrow \infty
$$

Then with (28),

$$
\begin{aligned}
E \int_{0}^{T}\left|Z_{s}^{n}-Z_{s}^{p}\right|^{2} d s & \leq 2\left(E\left(\sup _{0 \leq t \leq T}\left(L_{s}^{n}-L_{s}^{p}\right)^{2}\right)^{\frac{1}{2}}\left(E\left[\left(K_{T}^{n,+}\right)^{2}\right]\right)^{\frac{1}{2}}\right. \\
& \leq C\left(E\left(\sup _{0 \leq t \leq T}\left(L_{s}^{n}-L_{s}^{p}\right)^{2}\right]\right)^{\frac{1}{2}} \rightarrow 0
\end{aligned}
$$

as $n, p \rightarrow \infty$, i.e. $\left\{Z^{n}\right\}$ is a Cauchy sequence in the space $\mathbf{H}_{d}^{2}(0, T)$, and there exists a process $Z \in \mathbf{H}_{d}^{2}(0, T)$, s.t. as $n \rightarrow \infty$,

$$
E \int_{0}^{T}\left|Z_{s}^{n}-Z_{s}\right|^{2} d s \rightarrow 0 .
$$

Furthermore from Itô's formula, we have

$$
\begin{aligned}
& \sup _{0 \leq t \leq T}\left|Y_{t}^{n}-Y_{t}^{p}\right|^{2} \\
\leq 2 & \sup _{0 \leq t \leq T} \int_{t}^{T}\left(L_{s}^{n}-L_{s}^{p}\right) d\left(K_{s}^{n,+}-K_{s}^{p,+}\right)+2 \sup _{0 \leq t \leq T}\left|\int_{t}^{T}\left(Y_{s}^{n}-Y_{s}^{p}\right)\left(Z_{s}^{n}-Z_{s}^{p}\right) d B_{s}\right| .
\end{aligned}
$$

Taking the expectation on the both sides, by BDG inequality and (29), we get

$$
\begin{aligned}
& E \sup _{0 \leq t \leq T}\left|Y_{t}^{n}-Y_{t}^{p}\right|^{2} \\
\leq & 2 E\left[\int_{0}^{T}\left(L_{s}^{n}-L_{s}^{p}\right) d K_{s}^{n,+}\right]+C E \int_{0}^{T}\left(Y_{s}^{n}-Y_{s}^{p}\right)^{2}\left(Z_{s}^{n}-Z_{s}^{p}\right)^{2} d s \\
\leq & C\left(E\left[\sup _{0 \leq t \leq T}\left(L_{s}^{n}-L_{s}^{p}\right)^{2}\right]\right)^{\frac{1}{2}}+\frac{1}{2} E \sup _{0 \leq t \leq T}\left|Y_{s}^{n}-Y_{s}^{p}\right|^{2}+C E \int_{0}^{T}\left|Z_{s}^{n}-Z_{s}^{p}\right|^{2} d s .
\end{aligned}
$$

Hence, by (31) and (30), as $n, p \rightarrow \infty$,

$$
E \sup _{0 \leq t \leq T}\left|Y_{t}^{n}-Y_{t}^{p}\right|^{2} \rightarrow 0
$$

i.e. $\left\{Y^{n}\right\}$ is a Cauchy sequence in the space $\mathbf{S}^{2}(0, T)$, which implies that there exists a process $Y \in \mathbf{S}^{2}(0, T)$, s.t. as $n \rightarrow \infty$,

$$
E \sup _{0 \leq t \leq T}\left|Y_{t}^{n}-Y_{t}\right|^{2} \rightarrow 0
$$

Moreover, since $f$ is continuous and decreasing on $y$, with $Y_{t}^{n} \nearrow Y_{t}$,

$$
f\left(t, Y_{t}^{n}\right)-f\left(t, Y_{t}\right) \searrow 0,0 \leq t \leq T .
$$

By the monotonic limit theorem, we get $\int_{0}^{T}\left[f\left(t, Y_{t}^{n}\right)-f\left(t, Y_{t}\right)\right] d t \searrow 0$, and with (26) , it follows $E\left[\left(\int_{0}^{T} f\left(t, Y_{t}\right) d t\right)^{2}\right] \leq C$, then

$$
E\left[\left(\int_{0}^{T}\left(f_{n}\left(t, Y_{t}^{n}\right)-f\left(t, Y_{t}\right)\right) d t\right)^{2}\right] \rightarrow 0
$$


as $n \rightarrow \infty$.

From corollary [3.1, we know that for $\forall t \in[0, T], K_{t}^{n,-}$ is increasing with respect to $n$, and with $E\left[\left(K_{t}^{n,-}\right)^{2}\right] \leq C$, there exists $K_{t}^{-}$such that $K_{t}^{n,-} \nearrow K_{t}^{-}$in $\mathbf{L}^{2}\left(\mathcal{F}_{t}\right)$. Since for each $t \in[0, T]$, $E\left[\left(K_{t}^{n,+}\right)^{2}\right] \leq C$, the sequence $\left(K_{t}^{n,+}\right)$ has weak limit $K_{t}^{+}$in $\mathbf{L}^{2}\left(\mathcal{F}_{t}\right)$, with $E\left[\left(K_{t}^{+}\right)^{2}\right] \leq C$. Then for $0 \leq t \leq T,\left(Y, Z, K^{+}, K^{-}\right)$satisfies

$$
Y_{t}=\xi+\int_{t}^{T} f\left(s, Y_{s}\right) d s+K_{T}^{+}-K_{t}^{+}-\left(K_{T}^{-}-K_{t}^{-}\right)-\int_{t}^{T} Z_{s} d B_{s} .
$$

We will then prove that the convergence of $\left\{K^{n,+}\right\}$ and $\left\{K^{n,-}\right\}$ also holds in strong sense. First, we consider $\left\{K^{n,-}\right\}$, for $n, p \in \mathbf{N}$, with $n \geq p$, since $L_{t}^{n} \geq L_{t}^{p}$, by corollary 3.1, we have for $0 \leq s \leq t \leq T, K_{t}^{n,-}-K_{s}^{n,-} \geq K_{t}^{p,-}-K_{s}^{p,-}$. So $0 \leq K_{t}^{n,-}-K_{t}^{p,-} \leq K_{T}^{n,-}-K_{T}^{p,-}$, and it follows immediately by letting $n \rightarrow \infty$

$$
0 \leq K_{t}^{-}-K_{t}^{p,-} \leq K_{T}^{-}-K_{T}^{p,-}
$$

This inequality yields as $p \rightarrow \infty$,

$$
E \sup _{0 \leq t \leq T}\left|K_{t}^{-}-K_{t}^{p,-}\right|^{2} \leq E\left|K_{T}^{-}-K_{T}^{p,-}\right|^{2} \rightarrow 0
$$

Then we consider the term $\left\{K^{n,+}\right\}$. For this we rewrite (22) and (35) in the forward form:

$$
\begin{aligned}
K_{t}^{n,+} & =Y_{0}^{n}-Y_{t}^{n}-\int_{0}^{t} f\left(s, Y_{s}^{n}\right) d s+K_{t}^{n,-}+\int_{0}^{t} Z_{s}^{n} d B_{s} \\
K_{t}^{+} & =Y_{0}-Y_{t}-\int_{0}^{t} f\left(s, Y_{s}\right) d s+K_{t}^{-}+\int_{0}^{t} Z_{s} d B_{s}
\end{aligned}
$$

so consider the difference and take expectation on the both sides, by the BDG inequality, and $f\left(s, Y_{s}^{n}\right) \geq f\left(s, Y_{s}\right)$, it follows

$$
\begin{aligned}
E\left[\sup _{0 \leq t \leq T}\left|K_{t}^{n,+}-K_{t}^{+}\right|^{2}\right] \leq & 5\left|Y_{0}^{n}-Y_{0}\right|^{2}+5 E\left[\sup _{0 \leq t \leq T}\left|Y_{t}^{n}-Y_{t}\right|^{2}\right]+5 E\left(\int_{0}^{T}\left[f\left(s, Y_{s}^{n}\right)-f\left(s, Y_{s}\right)\right] d s\right)^{2} \\
& +5 E \sup _{0 \leq t \leq T}\left|K_{t}^{n,-}-K_{t}^{-}\right|^{2}+C E \int_{0}^{T}\left|Z_{s}^{n}-Z_{s}\right|^{2} d s .
\end{aligned}
$$

Then by (33), (34), (36) and (31), we deduce that

$$
E\left[\sup _{0 \leq t \leq T}\left|K_{t}^{n,+}-K_{t}^{+}\right|^{2}\right] \rightarrow 0
$$

The last thing to check is that (3) and (4) are also satisfied. Since for each $n \in \mathbf{N}, L_{t}^{n} \leq Y_{t}^{n} \leq U_{t}$, $0 \leq t \leq T$, with $Y_{t}^{n} \nearrow Y_{t}$ and $L_{t}^{n} \nearrow L_{t}$, then $L_{t} \leq Y_{t} \leq U_{t}$. From another part, the processes $K^{n,+}$ and $K^{n,-}$ are increasing, so the limit $K^{+}$and $K^{-}$are also increasing. Notice that $\left(Y^{n}, K^{n,+}, K^{n,-}\right)$ tends to $\left(Y, K^{+}, K^{-}\right)$uniformly in $t$ in probability, so the measure $d K^{n,+}$ (resp. $\left.d K^{n,-}\right)$ converges to $d K^{+}$(resp. $\left.d K^{-}\right)$weakly in probability. So

$$
\int_{0}^{T}\left(Y_{t}-L_{t}\right) d K_{t}^{n,+} \rightarrow \int_{0}^{T}\left(Y_{t}-L_{t}\right) d K_{t}^{+}, \int_{0}^{T}\left(Y_{t}^{n}-U_{t}\right) d K_{t}^{n,-} \rightarrow \int_{0}^{T}\left(Y_{t}-U_{t}\right) d K_{t}^{-}
$$


in probability as $n \rightarrow \infty$. Obviously $\int_{0}^{T}\left(Y_{t}-U_{t}\right) d K_{t}^{-} \leq 0$. On the other hand, for each $n \in \mathbf{N}$, $\int_{0}^{T}\left(Y_{t}^{n}-U_{t}\right) d K_{t}^{n,-}=0$. Hence

$$
\int_{0}^{T}\left(Y_{t}-U_{t}\right) d K_{t}^{-}=0, \text { a.s. }
$$

For the lower barrier, since $L^{n}$ converges to $L$ in $\mathbf{S}^{2}$, as $n \rightarrow \infty$, we have

$$
\begin{aligned}
& E \int_{0}^{T}\left(Y_{t}^{n}-L_{t}^{n}\right) d K_{t}^{n,+}-E \int_{0}^{T}\left(Y_{t}-L_{t}\right) d K_{t}^{+} \\
= & E \int_{0}^{T}\left(Y_{t}^{n}-Y_{t}\right) d K_{t}^{n,+}+E \int_{0}^{T}\left(Y_{t}-L_{t}\right) d\left(K_{t}^{n,+}-K_{t}^{+}\right)+E \int_{0}^{T}\left(L_{t}-L_{t}^{n}\right) d K_{t}^{n,+} \\
\leq & C\left(E\left[\sup _{0 \leq t \leq T}\left(Y_{t}^{n}-Y_{t}\right)^{2}\right]\right)^{\frac{1}{2}}+E \int_{0}^{T}\left(Y_{t}-L_{t}\right) d\left(K_{t}^{n,+}-K_{t}^{+}\right)+C\left(E\left[\sup _{0 \leq t \leq T}\left(L_{t}-L_{t}^{n}\right)^{2}\right]\right)^{\frac{1}{2}} \\
\rightarrow & 0 .
\end{aligned}
$$

Since $Y_{t} \geq L_{t}$, then $\int_{0}^{T}\left(Y_{t}-L_{t}\right) d K_{t}^{+} \geq 0$, while $E \int_{0}^{T}\left(Y_{t}^{n}-L_{t}^{n}\right) d K_{t}^{n,+}=0$, so $E \int_{0}^{T}\left(Y_{t}-L_{t}\right) d K_{t}^{+}=0$, then $\int_{0}^{T}\left(Y_{t}-L_{t}\right) d K_{t}^{+}=0$

Step 3. In this step, we study the general case for $L$ and $U$, when assumption 2.3' is satisfied:

$$
E\left[\varphi^{2}\left(\sup _{0 \leq t \leq T}\left(L_{t}\right)^{+}\right)\right]+E\left[\varphi^{2}\left(\sup _{0 \leq t \leq T}\left(U_{t}\right)^{-}\right)\right]<\infty,
$$

$L^{+}, U^{-} \in \mathbf{S}^{2}(0, T)$. But we still assume that for some $C>0$,

$$
|\xi|+\sup _{0 \leq t \leq T}|f(t, 0)| \leq C .
$$

For $n \in \mathbf{N}$, set $U^{n}=U \vee(-n)$; then $\sup _{0 \leq t \leq T}\left(U_{t}^{n}\right)^{-} \leq n$ and $U^{n} \geq U$, so assumption 2.3'(ii), (iii) are satisfied, and by the step 2, we know that there exists a triple $\left(Y^{n}, Z^{n}, K^{n}\right)$, with $K^{n}=K^{n,+}-K^{n,-}$, which satisfies

$$
Y_{t}^{n}=\xi+\int_{t}^{T} f\left(s, Y_{s}^{n}\right) d s+K_{T}^{n,+}-K_{t}^{n,+}-\left(K_{T}^{n,-}-K_{t}^{n,-}\right)-\int_{t}^{T} Z_{s}^{n} d B_{s},
$$

$L_{t} \leq Y_{t}^{n} \leq U_{t}^{n}, 0 \leq t \leq T$, and $\int_{0}^{T}\left(Y_{t}^{n}-L_{t}\right) d K_{t}^{n,+}=\int_{0}^{T}\left(Y_{t}^{n}-U_{t}^{n}\right) d K_{t}^{n,-}=0$.

Like in step 2, we consider the solution $(\bar{Y}, \bar{Z}, \bar{K})$ of the one lower barrier $\operatorname{RBSDE}(\xi, f, L)$ and the solution $(\underline{Y}, \underline{Z}, \underline{K})$ of the one super barrier $\operatorname{RBSDE}(\xi, f, U)$. Then by the comparison theorem 3.3, it follows that $\underline{Y}_{t} \leq Y_{t}^{n} \leq \bar{Y}_{t}, 0 \leq t \leq T$. So

$$
E\left[\sup _{0 \leq t \leq T}\left|Y_{t}^{n}\right|^{2}\right] \leq \max \left\{E\left[\sup _{0 \leq t \leq T}\left|\bar{Y}_{t}\right|^{2}\right], E\left[\sup _{0 \leq t \leq T}\left|\underline{Y}_{t}\right|^{2}\right]\right\} \leq C .
$$

Since $U_{t}^{n+1} \leq U_{t}^{n}, 0 \leq t \leq T$, thanks to the comparison theorem 3.3, $Y_{t}^{n} \searrow Y_{t}, 0 \leq t \leq T$. From (39) and Fatou's lemma, we get

$$
E\left[\sup _{0 \leq t \leq T}\left(Y_{t}\right)^{2}\right] \leq C
$$

and

$$
E \int_{0}^{T}\left|Y_{t}^{n}-Y_{t}\right|^{2} d t \rightarrow 0, \text { as } n \rightarrow \infty,
$$

which follows from the dominated convergence theorem. 
Notice that $f$ is decreasing on $y$, then $f\left(t, \bar{Y}_{t}\right) \leq f\left(t, Y_{t}^{n}\right) \leq f\left(t, \underline{Y}_{t}\right), 0 \leq t \leq T$, and with the integral property of $\underline{Y}$ and $\bar{Y}$, we have

$$
E\left[\left(\int_{0}^{t} f\left(s, Y_{s}^{n}\right) d s\right)^{2}\right] \leq \max \left\{E\left[\left(\int_{0}^{t} f\left(s, \bar{Y}_{s}\right) d s\right)^{2}\right], E\left[\left(\int_{0}^{t} f\left(s, \underline{Y}_{s}\right) d s\right)^{2}\right]\right\} \leq C .
$$

Then we use again the comparison theorem for the estimation of $K_{t}^{n,+}$. Consider the linear $\operatorname{RBSDE}\left(\xi, f\left(s, U_{s}^{+}\right), L, U\right)$, by results of [4, we know that there exists $\left(\widetilde{Y}, \widetilde{Z}, \widetilde{K}^{+}, \widetilde{K}^{-}\right) \in \mathbf{S}^{2}(0, T) \times$ $\mathbf{H}_{d}^{2}(0, T) \times \mathbf{A}^{2}(0, T) \times \mathbf{A}^{2}(0, T)$ satisfying the following:

$$
\begin{aligned}
& \widetilde{Y}_{t}=\xi+\int_{t}^{T} f\left(s,\left(U_{s}\right)^{+}\right) d s+\widetilde{K}_{T}^{+}-\widetilde{K}_{t}^{+}-\left(\widetilde{K}_{T}^{-}-\widetilde{K}_{t}^{-}\right)-\int_{t}^{T} \widetilde{Z}_{s} d B_{s} \\
& L_{t} \leq \widetilde{Y}_{t} \leq U_{t}, \int_{0}^{T}\left(\widetilde{Y}_{t}-L_{t}\right) d \widetilde{K}_{t}^{+}=\int_{0}^{T}\left(\widetilde{Y}_{t}-U_{t}\right) d \widetilde{K}_{t}^{-}=0 .
\end{aligned}
$$

We admits for a instant the following lemma, which will be proved later.

Lemma 2.4 For $0 \leq s \leq t \leq T, K_{t}^{n,+}-K_{s}^{n,+} \leq \widetilde{K}_{t}^{+}-\widetilde{K}_{s}^{+}$, and $K_{T}^{n,+} \leq \widetilde{K}_{T}^{+}$.

Now we have

$$
E\left[\left(K_{T}^{n,+}\right)^{2}\right] \leq E\left[\left(\widetilde{K}_{T}^{+}\right)^{2}\right] \leq C,
$$

Then apply the Itô's formula to $\left|Y_{t}^{n}\right|^{2}$ on the interval $[t, T]$, by the same method as in step 2, we have the following estimates

$$
E\left[\left(K_{T}^{n,-}\right)^{2}\right]+E \int_{0}^{T}\left|Z_{s}^{n}\right|^{2} d s \leq C .
$$

Since $U_{t}^{n}-U_{t} \downarrow 0$, for each $t \in[0, T]$, and $U_{t}^{n}-U_{t}$ is continuous, by the Dini's theorem again, the convergence holds uniformly on the interval $[0, T]$, i.e.

$$
E\left[\sup _{0 \leq t \leq T}\left(U_{t}^{n}-U_{t}\right)^{2}\right] \rightarrow 0, \text { as } n \rightarrow \infty .
$$

Now we are in the same situation as step 2. With the same arguments, we deduce that there exists processes $Y \in \mathbf{S}^{2}(0, T), Z \in \mathbf{H}_{d}^{2}(0, T), K^{+} \in \mathbf{A}^{2}(0, T), K^{-} \in \mathbf{A}^{2}(0, T)$, s.t. as $n \rightarrow \infty$,

$$
E\left[\sup _{0 \leq t \leq T}\left|Y_{t}^{n}-Y_{t}\right|+\int_{0}^{T}\left|Z_{s}^{n}-Z_{s}\right|^{2} d s+\sup _{0 \leq t \leq T}\left|K_{t}^{n,+}-K_{t}^{+}\right|^{2}+\sup _{0 \leq t \leq T}\left|K_{t}^{n,-}-K_{t}^{-}\right|^{2}\right] \rightarrow 0,
$$

which satisfies

$$
Y_{t}=\xi+\int_{t}^{T} f\left(s, Y_{s}\right) d s+K_{T}^{+}-K_{t}^{+}-\left(K_{T}^{-}-K_{t}^{-}\right)-\int_{t}^{T} Z_{s} d B_{s}
$$

The last thing to check is that (3) and (4) of definition 2.1 are satisfied. Since for each $n \in \mathbf{N}$, $L_{t} \leq Y_{t}^{n} \leq U_{t}^{n}, 0 \leq t \leq T$, with $Y_{t}^{n} \searrow Y_{t}$ and $U_{t}^{n} \searrow U_{t}$, then $L_{t} \leq Y_{t} \leq U_{t}$. On the other hand, the processes $K^{n,+}$ and $K^{n,-}$ are increasing, so the limit $K^{+}$and $K^{-}$are also increasing. Notice that $\left(Y^{n}, K^{n,+}, K^{n,-}\right)$ tends to $\left(Y, K^{+}, K^{-}\right)$uniformly in $t$ in probability, and $U^{n}$ converges to $U$ in $\mathbf{S}^{2}$, as $n \rightarrow \infty$, similarly as step 2 , we get

$$
\int_{0}^{T}\left(Y_{t}-K_{t}\right) d K_{t}^{+}=\int_{0}^{T}\left(Y_{t}-U_{t}\right) d K_{t}^{-}=0, \text { a.s. }
$$


Step 4. In this step, we will partly relax the bounded assumption for $\xi$ and $f(t, 0)$. We only suppose that for a constant $c$,

$$
\xi \geq c \text { and } \inf _{0 \leq t \leq T} f(t, 0) \geq c .
$$

We approximate $\xi$ and $f(t, 0)$ by a sequence whose elements satisfy the bounded assumption in step 3, as following: for $n \in \mathbf{N}$, set

$$
\xi_{n}=\xi \wedge n, f_{n}(t, y)=f(t, y)-f(t, 0)+f(t, 0) \wedge n .
$$

Obviously, $\left(\xi^{n}, f^{n}\right)$ satisfies the assumptions of the step 3 , and since $\xi \in \mathbf{L}^{2}\left(\mathcal{F}_{T}\right), f(t, 0) \in \mathbf{H}^{2}(0, T)$, then

$$
E\left[\left|\xi^{n}-\xi\right|^{2}\right] \rightarrow 0, E \int_{0}^{T}\left|f(t, 0)-f_{n}(t, 0)\right|^{2} \rightarrow 0
$$

as $n \rightarrow \infty$.

From the results in step 3 , for each $n \in \mathbf{N}$, there exists $\left(Y_{t}^{n}, Z_{t}^{n}, K_{t}^{n}\right)_{0 \leq t \leq T} \in \mathbf{S}^{2}(0, T) \times$ $\mathbf{H}_{d}^{2}(0, T) \times \mathbf{V} \mathbf{F}^{2}(0, T)$, with $K^{n}=K^{n,+}-K^{n,-}$, which is the unique solution of the $\operatorname{RBSDE}\left(\xi^{n}, f_{n}, L, U\right)$, i.e.

$$
\begin{aligned}
Y_{t}^{n} & =\xi^{n}+\int_{t}^{T} f_{n}\left(s, Y_{s}^{n}\right) d s+K_{T}^{n,+}-K_{t}^{n,+}-\left(K_{T}^{n,-}-K_{t}^{n,-}\right)-\int_{t}^{T} Z_{s}^{n} d B_{s}, \\
L_{t} & \leq Y_{t}^{n} \leq U_{t}, \int_{0}^{T}\left(Y_{t}^{n}-L_{t}\right) d K_{t}^{n,+}=\int_{0}^{T}\left(Y_{t}^{n}-U_{t}\right) d K_{t}^{n,-}=0 .
\end{aligned}
$$

Like in step 3, we consider the solution $(\bar{Y}, \bar{Z}, \bar{K})$ of one lower barrier $\operatorname{RBSDE}(\xi, f, L)$ and the solution $(\underline{Y}, \underline{Z}, \underline{K})$ of one super barrier $\operatorname{RBSDE}\left(\xi^{-}, \underline{f}, U\right)$, where $\xi^{-}$is the negative part of $\xi, f(t, y)=$ $f(t, y)-f(t, 0)+(f(t, 0))^{-}$. Then we can take the $\operatorname{RBSDE}(\xi, f, L)\left(\operatorname{resp} \cdot \operatorname{RBSDE}\left(\xi^{-}, \bar{f}, U\right)\right)$ as a RBSDE with two barriers associated to the parameters $(\xi, f, L, \bar{U})\left(\operatorname{resp} .\left(\xi^{-}, \underline{f}, \underline{L}, U\right)\right)$, where $\bar{U}=\infty$ and $\underline{L}=-\infty$. By the comparison theorem $[3.3$, since

$$
\xi \geq \xi^{n} \geq \xi^{-}, f(t, y) \geq f_{n}(t, y) \geq \underline{f}(t, y)
$$

it follows that

$$
\underline{Y}_{t} \leq Y_{t}^{n} \leq \bar{Y}_{t}, 0 \leq t \leq T
$$

So

$$
E\left[\sup _{0 \leq t \leq T}\left|Y_{t}^{n}\right|^{2}\right] \leq \max \left\{E \left[\sup _{0 \leq t \leq T}\left|\bar{Y}_{t}\right|^{2}, E\left[\sup _{0 \leq t \leq T}\left|\underline{Y}_{t}\right|^{2}\right\} \leq C .\right.\right.
$$

Then by the comparison theorem [3.6, since for all $(s, y) \in[0, T] \times \mathbb{R}, n \in \mathbf{N}, \xi_{1} \leq \xi_{n}, f_{1}(s, y) \leq$ $f_{n}(s, y)$, we have $K_{t}^{1,+} \geq K_{t}^{n,+} \geq 0$ for $0 \leq t \leq T$, so $E\left[\left(K_{t}^{n,+}\right)^{2}\right] \leq E\left[\left(K_{t}^{1,+}\right)^{2}\right] \leq C$. Following the same steps, we deduce that

$$
\left.E\left[\int_{0}^{t} f\left(s, Y_{s}^{n}\right) d s\right)^{2}\right]+E \int_{0}^{T}\left|Z_{s}^{n}\right|^{2} d s+E\left[\left(K_{t}^{n,-}\right)^{2}\right]+E\left[\left(K_{t}^{n,+}\right)^{2}\right] \leq C .
$$

Due to the comparison theorem [3.3, since for all $(s, y) \in[0, T] \times \mathbb{R}, n \in \mathbf{N}, \xi_{n} \leq \xi_{n+1}, f_{n}(s, y) \leq$ $f_{n+1}(s, y)$, we have $Y_{t}^{n} \leq Y_{t}^{n+1}, 0 \leq t \leq T$, a.s. Hence

$$
Y_{t}^{n} \nearrow Y_{t}, 0 \leq t \leq T \text {. a.s. }
$$


Applying Itô formula to $\left|Y_{t}^{n}-Y_{t}^{p}\right|^{2}$, for $n, p \in \mathbf{N}, n \geq p$, on $[t, T]$, we get

$$
\begin{aligned}
& E\left|Y_{t}^{n}-Y_{t}^{p}\right|^{2}+E \int_{t}^{T}\left|Z_{s}^{n}-Z_{s}^{p}\right|^{2} d s \\
\leq & E\left|\xi^{n}-\xi^{p}\right|^{2}+E \int_{t}^{T}\left|Y_{s}^{n}-Y_{s}^{p}\right|^{2} d s+E \int_{t}^{T}\left|f_{n}(s, 0)-f_{p}(s, 0)\right|^{2} d s,
\end{aligned}
$$

since $\int_{t}^{T}\left(Y_{s}^{n}-Y_{s}^{p}\right) d\left(K_{s}^{n,+}-K_{s}^{p,+}\right)-\int_{t}^{T}\left(Y_{s}^{n}-Y_{s}^{p}\right) d\left(K_{s}^{n,-}-K_{s}^{p,-}\right) \leq 0$. Hence from Gronwall's inequality and (47), we deduce

$$
\sup _{0 \leq t \leq T} E\left|Y_{t}^{n}-Y_{t}^{p}\right|^{2} \rightarrow 0, \quad E \int_{0}^{T}\left|Z_{s}^{n}-Z_{s}^{p}\right|^{2} d s \rightarrow 0 .
$$

Consequently there exists $\left(Z_{t}\right)_{0 \leq t \leq T} \in \mathbf{H}_{d}^{2}(0, T)$, s.t.

$$
E \int_{0}^{T}\left|Z_{s}^{n}-Z_{s}\right|^{2} d s \rightarrow 0 .
$$

Using again Itô formula, taking sup and the expectation, in view of the BDG inequality, $Y_{t}^{n} \geq$ $Y_{t}^{p}$, assumption 2.4-(iii) and $f_{n}(t, 0) \geq f_{p}(t, 0)$, we get

$$
\begin{aligned}
E\left[\sup _{0 \leq t \leq T}\left|Y_{t}^{n}-Y_{t}^{p}\right|^{2}\right] \leq & E\left|\xi^{n}-\xi^{p}\right|+4 T E \int_{0}^{T}\left|f_{n}(s, 0)-f_{p}(s, 0)\right|^{2} d s+\frac{1}{4} E \sup _{0 \leq t \leq T}\left|Y_{s}^{n}-Y_{s}^{p}\right|^{2} \\
& +\frac{1}{4} E\left[\sup _{0 \leq t \leq T}\left|Y_{t}^{n}-Y_{t}^{p}\right|^{2}\right]+c E \int_{0}^{T}\left|Z_{s}^{n}-Z_{s}^{p}\right|^{2} d s .
\end{aligned}
$$

From (47) and (151), it follows $E\left[\sup _{0 \leq t \leq T}\left|Y_{t}^{n}-Y_{t}^{p}\right|^{2}\right] \rightarrow 0$, as $n, p \rightarrow \infty$, i.e. the sequence $\left\{Y^{n}\right\}$ is a Cauchy sequence in the space $\mathbf{S}^{2}(0, T)$. Consequently, with (50), we have $Y \in \mathbf{S}^{2}(0, T)$ and

$$
E\left[\sup _{0 \leq t \leq T}\left|Y_{t}^{n}-Y_{t}\right|^{2}\right] \rightarrow 0 .
$$

By the comparison theorem [3.6, since for all $(s, y) \in[0, T] \times \mathbb{R}, n \in \mathbf{N}, \xi_{n} \leq \xi_{n+1}, f_{n}(s, y) \leq$ $f_{n+1}(s, y)$, we have $K_{t}^{n,+} \geq K_{t}^{n+1,+} \geq 0$, and $0 \leq K_{t}^{n,-} \leq K_{t}^{n+1,-}$ for $0 \leq t \leq T$, so

$$
K_{t}^{n,+} \searrow K_{t}^{+}, K_{t}^{n,-} \nearrow K_{t}^{-}
$$

with (49), by the monotonic limit theorem, it follows that $K_{t}^{n,+} \rightarrow K_{t}^{+}, K_{t}^{n,-} \rightarrow K_{t}^{-}$in $\mathbf{L}^{2}\left(\mathcal{F}_{t}\right)$, and $E\left[\left(K_{t}^{+}\right)^{2}+\left(K_{t}^{-}\right)^{2}\right]<\infty$, moreover, $\left(K_{t}^{+}\right)_{0 \leq t \leq T}$ and $\left(K_{t}^{-}\right)_{0 \leq t \leq T}$ are increasing.

Notice that since $f(t, y)$ is decreasing and continuous in $y$, and $Y_{t}^{n} \nearrow Y_{t}$, we have $f\left(t, Y_{t}^{n}\right) \searrow$ $f\left(t, Y_{t}\right)$. Then by the monotonic limit theorem, $\int_{0}^{t} f\left(s, Y_{s}^{n}\right) d s \searrow \int_{0}^{t} f\left(s, Y_{s}\right) d s$. With (49)), it follows that $\int_{0}^{t} f\left(s, Y_{s}^{n}\right) d s \rightarrow \int_{0}^{t} f\left(s, Y_{s}\right) d s$ in $\mathbf{L}^{2}\left(\mathcal{F}_{t}\right)$, as $n \rightarrow \infty$.

Now we need to prove that the convergence of $\left\{K^{n,+}\right\}$ and $\left\{K^{n,-}\right\}$ holds in a stronger sense. Using again the comparison theorem [3.6. since for all $(s, y) \in[0, T] \times \mathbb{R}, n, p \in \mathbf{N}$, with $n \geq p$, $\xi_{p} \leq \xi_{n}, f_{p}(s, y) \leq f_{n}(s, y)$, we have for $0 \leq s \leq t \leq T$,

$$
K_{t}^{p,+}-K_{s}^{p,+} \geq K_{t}^{n,+}-K_{s}^{n,+} \geq 0,
$$

Then let $n \rightarrow \infty$, for $t \in[0, T], K_{T}^{p,+}-K_{T}^{+} \geq K_{t}^{p,+}-K_{t}^{+} \geq 0$. So as $n \rightarrow 0$,

$$
E \sup _{0 \leq t \leq T}\left|K_{t}^{p,+}-K_{t}^{+}\right|^{2} \leq E\left|K_{T}^{p,+}-K_{T}^{+}\right|^{2} \rightarrow 0
$$


Similarly, we have $E \sup _{0 \leq t \leq T}\left|K_{t}^{-}-K_{t}^{p,-}\right|^{2} \leq E\left|K_{T}^{-}-K_{T}^{p,-}\right|^{2} \rightarrow 0$.

It remains to check if $\left(Y_{t}, Z_{t}, K_{t}\right)_{0 \leq t \leq T}$ satisfies (3) and (4) of the definition 2.1. Since $L_{t} \leq$ $Y_{t}^{n} \leq U_{t}, 0 \leq t \leq T$, then letting $n \rightarrow \infty, L_{t} \leq Y_{t} \leq U_{t}, 0 \leq t \leq T$, a.s.. Furthermore $\left(Y^{n}, K^{n,+}\right)$ tends to $\left(Y, K^{+}\right)$uniformly in $t$ in probability, as $n \rightarrow \infty$, then the measure $d K^{n,+} \rightarrow d K^{+}$weakly in probability, as $n \rightarrow \infty$, i.e. $\int_{0}^{T}\left(Y_{t}^{n}-L_{t}\right) d K_{t}^{n,+} \rightarrow \int_{0}^{T}\left(Y_{t}-L_{t}\right) d K_{t}^{+}$, in probability. While $L_{t} \leq Y_{t} \leq U_{t}, 0 \leq t \leq T$, so $\int_{0}^{T}\left(Y_{t}-L_{t}\right) d K_{t}^{+} \geq 0$. On the other hand $\int_{0}^{T}\left(Y_{t}^{n}-L_{t}\right) d K_{t}^{n,+}=0$, so $\int_{0}^{T}\left(Y_{t}-L_{t}\right) d K_{t}^{+}=0$. Similarly, $\int_{0}^{T}\left(Y_{t}-U_{t}\right) d K_{t}^{-}=0$, i.e. the triple $\left(Y_{t}, Z_{t}, K_{t}\right)_{0 \leq t \leq T}$ is the solution of $\operatorname{RBSDE}(\xi, f, L)$, under the assumption (46).

Step 5. Now we consider a terminal condition $\xi \in \mathbf{L}^{2}\left(\mathcal{F}_{T}\right)$ and a coefficient $f$ which satisfies assumption 2.4. For $n \in \mathbf{N}$, set

$$
\xi_{n}=\xi \vee(-n), f_{n}(t, y)=f(t, y)-f(t, 0)+f(t, 0) \vee(-n) .
$$

Obviously, $\left(\xi^{n}, f^{n}\right)$ satisfies the assumptions of the step 4 , and since $\xi \in \mathbf{L}^{2}\left(\mathcal{F}_{T}\right), f(t, 0) \in \mathbf{H}^{2}(0, T)$, then

as $n \rightarrow \infty$.

$$
E\left[\left|\xi^{n}-\xi\right|^{2}\right] \rightarrow 0, E \int_{0}^{T}\left|f(t, 0)-f_{n}(t, 0)\right|^{2} \rightarrow 0
$$

From the results in step 3 , for each $n \in \mathbf{N}$, there exists $\left(Y_{t}^{n}, Z_{t}^{n}, K_{t}^{n}\right)_{0 \leq t \leq T} \in \mathbf{S}^{2}(0, T) \times$ $\mathbf{H}_{d}^{2}(0, T) \times \mathbf{V} \mathbf{F}^{2}(0, T)$, with $K^{n}=K^{n,+}-K^{n,-}$, which is the unique solution of the $\operatorname{RBSDE}\left(\xi^{n}, f_{n}, L, U\right)$. Like in step 4 , we consider the solution $(\bar{Y}, \bar{Z}, \bar{K})$ of the one lower barrier $\operatorname{RBSDE}\left(\xi^{+}, \bar{f}, L\right)$, where $\xi^{+}$is the positive part of $\xi, \bar{f}(t, y)=f(t, y)-f(t, 0)+(f(t, 0))^{+}$, and the solution $(\underline{Y}, \underline{Z}, \underline{K})$ of the one super barrier $\operatorname{RBSDE}(\xi, f, U)$. Then we can take the $\operatorname{RBSDE}\left(\xi^{+}, \bar{f}, L\right)(\operatorname{resp} . \operatorname{RBSDE}(\xi, f, U))$ as a RBSDE with two barriers associated to the parameters $\left(\xi^{+}, \bar{f}, L, \bar{U}\right)(\operatorname{resp} .(\xi, f, \underline{L}, U))$, where $\bar{U}=\infty$ and $\underline{L}=-\infty$. Thanks to the comparison theorem 3.3 , we have that

$$
E\left[\sup _{0 \leq t \leq T}\left|Y_{t}^{n}\right|^{2}\right] \leq \max \left\{E \left[\sup _{0 \leq t \leq T}\left|\bar{Y}_{t}\right|^{2}, E\left[\sup _{0 \leq t \leq T}\left|\underline{Y}_{t}\right|^{2}\right\} \leq C .\right.\right.
$$

For $n, p \in \mathbf{N}$, with $n \geq p$, we have $\xi^{n} \leq \xi^{p}$ and $f_{n}(t, y) \leq f_{p}(t, y), \forall(t, y) \in[0, T] \times \mathbb{R}$. From approximations for $\xi^{n}, \xi^{p}, f_{n}(t, y)$ and $f_{p}(t, y)$ as following:

$$
\begin{aligned}
\xi^{n, m} & :=\xi^{n} \wedge m, \xi^{p, m}:=\xi^{p} \wedge m \\
f_{n, m}(t, y) & =f_{n}(t, y)-f_{n}(t, 0)+f_{n}(t, 0) \wedge m=f(t, y)-f(t, 0)+(f(t, 0) \vee(-n)) \wedge m, \\
f_{p, m}(t, y) & =f_{p}(t, y)-f_{p}(t, 0)+f_{p}(t, 0) \wedge m=f(t, y)-f(t, 0)+(f(t, 0) \vee(-p)) \wedge m,
\end{aligned}
$$

then the parameters satisfy the assumptions in theorem [3.6, and

$$
\xi^{n, m} \leq \xi^{p, m}, f_{n, m}(t, y) \leq f_{p, m}(t, y) .
$$

Consider the solution $\left(Y^{n, m}, Z^{n, m}, K^{n, m}\right)\left(\right.$ resp. $\left.\left(Y^{p, m}, Z^{p, m}, K^{p, m}\right)\right)$ of the $\operatorname{RBSDE}\left(\xi^{n, m}, f_{n, m}, L, U\right)($ resp. $\left.\left(\xi^{p, m}, f_{p, m}, L, U\right)\right)$; by the comparison theorem [3.6. for $0 \leq s \leq t \leq T$, we have $K_{t}^{n, m,-}-K_{s}^{n, m,-} \leq$ $K_{t}^{p, m,-}-K_{s}^{p, m,-}$. Then by the convergence results in step 4 , let $m \rightarrow \infty$, we get

$$
K_{t}^{n,-}-K_{s}^{n,-} \leq K_{t}^{p,-}-K_{s}^{p,-}, \text { for } n \geq p .
$$

So we have $0 \leq K_{t}^{n,-} \leq K_{t}^{1,-}$, then $E\left[\left(K_{t}^{n,-}\right)^{2}\right] \leq E\left[\left(K_{t}^{1,-}\right)^{2}\right] \leq C$. By the same method as previous step, we deduce that

$$
\left.E\left[\int_{0}^{t} f\left(s, Y_{s}^{n}\right) d s\right)^{2}\right]+E\left[\left(K_{T}^{n,+}\right)^{2}\right]+E \int_{0}^{T}\left|Z_{s}^{n}\right|^{2} d s \leq C .
$$


Now we are in the same situation as in step 4, and following the same method, we get that the sequence $\left(Y_{t}^{n}, Z_{t}^{n}, K_{t}^{n,+}, K_{t}^{n,-}\right)$ converge to $\left(Y_{t}, Z_{t}, K_{t}^{+}, K_{t}^{-}\right)$as $n \rightarrow \infty$, in $\mathbf{S}^{2}(0, T) \times \mathbf{H}_{d}^{2}(0, T) \times$ $\mathbf{A}^{2}(0, T) \times \mathbf{A}^{2}(0, T)$, and $\left(Y_{t}, Z_{t}, K_{t}^{+}, K_{t}^{-}\right)$is the solution to the $\operatorname{RBSDE}(\xi, f, L, U)$.

\section{Appendix}

\subsection{Proofs of Lemmas}

In this subsection, we prove lemma 2.3 and lemma 2.4, which play important roles in previous section.

Proof of Lemma 2.3; Obviously $f\left(s,\left(L_{s}\right)^{-}\right) \in \mathbf{H}^{2}(0, T)$, in view of assumption 2.3'. Consider for $m, n \in \mathbf{N}$, the following RBSDEs with one lower barrier,

$$
\begin{aligned}
& \tilde{Y}_{t}^{m}=\xi+\int_{t}^{T} f\left(s,\left(L_{s}\right)^{-}\right) d s-m \int_{t}^{T}\left(\widetilde{Y}_{s}^{m}-U_{s}\right)^{+} d s+\widetilde{K}_{T}^{m,+}-\widetilde{K}_{t}^{m,+}-\int_{t}^{T} \widetilde{Z}_{s}^{m} d B_{s}, \\
& \tilde{Y}_{t}^{m} \geq L_{t}, \int_{0}^{T}\left(\widetilde{Y}_{t}^{m}-L_{t}\right) d \widetilde{K}_{t}^{m,+}=0,
\end{aligned}
$$

and

$$
\begin{aligned}
Y_{t}^{m, n} & =\xi+\int_{t}^{T} f\left(s, Y_{s}^{m, n}\right) d s-m \int_{t}^{T}\left(Y_{s}^{m, n}-U_{s}\right)^{+} d s+K_{T}^{m, n,+}-K_{t}^{m, n,+}-\int_{t}^{T} Z_{s}^{m, n} d B_{s}, \\
Y_{t}^{m, n} & \geq L_{t}^{n}, \int_{0}^{T}\left(Y_{t}^{m, n}-L_{t}^{n}\right) d K_{t}^{m, n,+}=0 .
\end{aligned}
$$

Since $Y_{t}^{m, n} \geq L_{t}^{n} \geq\left(L_{t}\right)^{-}$, we get $f\left(t, Y_{t}^{m, n}\right) \leq f\left(t, L_{t}^{n}\right) \leq f\left(t,\left(L_{t}\right)^{-}\right)$. Then for $m, n \in \mathbf{N}$, $\forall t \in[0, T]$

$$
f\left(t, Y_{t}^{m, n}\right)-m\left(Y_{t}^{m, n}-U_{t}\right)^{+} \leq f\left(t,\left(L_{t}\right)^{-}\right)-m\left(Y_{t}^{m, n}-U_{t}\right)^{+}, L_{t}^{n} \leq L_{t} .
$$

By the general comparison theorem for RBSDE with one barrier theorem 3.1 , it follows $Y_{t}^{m, n} \leq \widetilde{Y}_{t}^{m}$, $\forall t \in[0, T]$. Denote $\widetilde{K}_{t}^{m,-}=m \int_{0}^{t}\left(\widetilde{Y}_{s}^{m}-U_{s}\right)^{+} d s$ and $K_{t}^{m, n,-}=m \int_{0}^{t}\left(Y_{s}^{m, n}-U_{s}\right)^{+} d s$, then we get for $0 \leq s \leq t \leq T$

$$
K_{t}^{m, n,-}-K_{s}^{m, n,-} \leq \widetilde{K}_{t}^{m,-}-\widetilde{K}_{s}^{m,-} .
$$

Thanks to the convergence result in step 1 and in [12, notice that $\left(L^{n}\right)^{+}$is bounded, we know that as $m \rightarrow \infty, \widetilde{K}_{t}^{m,-} \rightarrow \widetilde{K}_{t}^{-}, K_{t}^{m, n,-} \rightarrow K_{t}^{n,-}$, in $\mathbf{L}^{2}\left(\mathcal{F}_{t}\right)$. Here $\widetilde{K}_{t}^{-}$and $K_{t}^{n,-}$ are increasing processes with respect to the upper barrier $U$ of the solution of the $\operatorname{RBSDE}\left(\xi, f\left(t,\left(L_{t}\right)^{-}\right), L, U\right)$ and $\operatorname{RBSDE}\left(\xi, f, L^{n}, U\right)$, respectively. Then from (55), we deduce that for $0 \leq s \leq t \leq T$,

$$
K_{t}^{n,-}-K_{s}^{n,-} \leq \widetilde{K}_{t}^{-}-\widetilde{K}_{s}^{-} .
$$

It follows immediately that $K_{T}^{n,-} \leq \widetilde{K}_{T}^{-}$.

Proof of Lemma 2.4: Obviously $f\left(s, U_{s}{ }^{+}\right) \in \mathbf{H}^{2}(0, T)$, in view of assumption 2.3'-(i). Consider the following RBSDEs with one barrier, for $n, m, p \in \mathbf{N}$, with $U^{n}=U \vee(-n)$,

$$
\begin{aligned}
& \tilde{Y}_{t}^{n, m, p}=\xi+\int_{t}^{T} f\left(s,\left(U_{s}\right)^{+}\right) d s+m \int_{t}^{T}\left(\widetilde{Y}_{s}^{n, m, p}-L_{s}^{p}\right)^{-} d s-\left(\widetilde{K}_{T}^{n, m, p,-}-\widetilde{K}_{t}^{n, m, p,-}\right)-\int_{t}^{T} \widetilde{Z}_{s}^{n, m, p} d B_{s}, \\
& \tilde{Y}_{t}^{n, m, p} \leq U_{t}^{n}, \int_{0}^{T}\left(\widetilde{Y}_{t}^{n, m, p}-U_{t}\right) d \widetilde{K}_{t}^{n, m, p,-}=0,
\end{aligned}
$$


and

$$
\begin{aligned}
Y_{t}^{n, m, p} & =\xi+\int_{t}^{T} f\left(s, Y_{s}^{n, m, p}\right) d s+m \int_{t}^{T}\left(Y_{s}^{n, m, p}-L_{s}^{p}\right)^{-} d s-\left(K_{T}^{n, m, p,-}-K_{t}^{n, m, p,-}\right)-\int_{t}^{T} Z_{s}^{n, m, p} d B_{s}, \\
Y_{t}^{n, m, p} & \leq U_{t}^{n}, \int_{0}^{T}\left(Y_{t}^{n, m, p}-U_{t}^{n}\right) d K_{t}^{n, m, p,-}=0 .
\end{aligned}
$$

Since $Y_{t}^{n, m, p} \leq U_{t}^{n} \leq\left(U_{t}\right)^{+}$, by monotonic property of $f$, we get $f\left(t, Y_{t}^{n, m, p}\right) \geq f\left(t,\left(U_{t}\right)^{+}\right)$. So

$$
f\left(t,\left(U_{t}\right)^{+}\right)+m\left(Y_{t}^{n, m, p}-L_{t}^{p}\right)^{-} \leq f\left(t, Y_{t}^{n, m, p}\right)+m\left(Y_{t}^{n, m, p}-L_{t}^{p}\right)^{-}, U_{t} \leq U_{t}^{n},
$$

from general comparison theorem for RBSDE with one barrier 3.1, we have $Y_{t}^{n, m, p} \geq \tilde{Y}_{t}^{n, m, p}$. Set $K_{t}^{n, m, p,+}=m \int_{0}^{t}\left(Y_{s}^{n, m, p}-L_{s}^{p}\right)^{-} d s, \widetilde{K}_{t}^{n, m, p,+}=m \int_{0}^{t}\left(\widetilde{Y}_{s}^{n, m, p}-L_{s}^{p}\right)^{-} d s$, then for $0 \leq s \leq t \leq T$

$$
K_{t}^{n, m, p,+}-K_{s}^{n, m, p,+} \leq \widetilde{K}_{t}^{n, m, p,+}-\widetilde{K}_{s}^{n, m, p,+} .
$$

Notice that $\left(L^{p}\right)^{+}$and $\left(U^{n}\right)^{-}$are bounded, by convergence results in step 1 , and the convergence result in [12], as $m \rightarrow \infty$, for $0 \leq s \leq t \leq T$, we have

$$
K_{t}^{n, p,+}-K_{s}^{n, p,+} \leq \widetilde{K}_{t}^{n, p,+}-\widetilde{K}_{s}^{n, p,+},
$$

where $K_{t}^{n, p,+}$ and $\widetilde{K}_{t}^{n, p,+}$ are the increasing processes corresponding to lower barrier $L^{p}$ for $\operatorname{RBSDE}\left(\xi, f, L^{p}, U^{n}\right)$ and $\operatorname{RBSDE}\left(\xi, f\left(t,\left(U_{t}\right)^{+}\right), L^{p}, U^{n}\right)$, respectively. Then thanks to the convergence result in step 2 for the approximation of lower barrier $L$, we have that as $p \rightarrow \infty$,

$$
K_{t}^{n, p,+} \rightarrow K_{t}^{n,+} \text { and } \widetilde{K}_{t}^{n, p,+} \rightarrow \widetilde{K}_{t}^{n,+} \text { in } \mathbf{L}^{2}\left(\mathcal{F}_{t}\right) .
$$

Here $K^{n,+}$ (resp. $\widetilde{K}^{n,+}$ ) is the increasing process corresponding to lower barrier $L$ for $\operatorname{RBSDE}\left(\xi, f, L, U^{n}\right)$ (resp. $\left.\operatorname{RBSDE}\left(\xi, f\left(t,\left(U_{t}\right)^{+}\right), L, U^{n}\right)\right)$. It follows for $0 \leq s \leq t \leq T$

$$
K_{t}^{n,+}-K_{s}^{n,+} \leq \widetilde{K}_{t}^{n,+}-\widetilde{K}_{s}^{n,+} .
$$

Finally by comparison theorem 3.2 , since $U_{t} \leq U_{t}^{n}, \forall t \in[0, T]$, we get

$$
\widetilde{K}_{t}^{+}-\widetilde{K}_{s}^{+} \geq \widetilde{K}_{t}^{n,+}-\widetilde{K}_{s}^{n,+},
$$

where $\widetilde{K}_{t}^{+}$is the increasing process corresponding to lower barrier $L$ for $\operatorname{RBSDE}\left(\xi, f\left(t,\left(U_{t}\right)^{+}\right), L, U^{n}\right)$.

So for $0 \leq s \leq t \leq T$

Specially, $K_{T}^{n,+} \leq \widetilde{K}_{T}^{+}$.

$$
K_{t}^{n,+}-K_{s}^{n,+} \leq \widetilde{K}_{t}^{+}-\widetilde{K}_{s}^{+}
$$

\subsection{Comparison theorems}

First we need a general comparison theorem for the RBSDE with one lower barrier.

Theorem 3.1 (General case for RBSDE's) Suppose that the parameters $\left(\xi^{1}, f^{1}, L^{1}\right)$ and $\left(\xi^{2}, f^{2}, L^{2}\right)$ satisfy assumption 2.1-2.3-(i). Let the triples $\left(Y^{1}, Z^{1}, K^{1}\right),\left(Y^{2}, Z^{2}, K^{2}\right)$ be respectively the solutions of the $\operatorname{RBSDE}\left(\xi^{1}, f^{1}, L^{1}\right)$ and $\operatorname{RBSDE}\left(\xi^{2}, f^{2}, L^{2}\right)$, i.e.

$$
Y_{t}^{i}=\xi^{i}+\int_{t}^{T} f^{i}\left(s, Y_{s}^{i}, Z_{s}^{i}\right) d s+K_{T}^{i}-K_{t}^{i}-\int_{t}^{T} Z_{s}^{i} d B_{s},
$$

$Y_{t}^{i} \geq L_{t}^{i}, 0 \leq t \leq T$, and $\int_{0}^{T}\left(Y_{s}^{i}-L_{s}^{i}\right) d K_{s}^{i}=0, i=1,2$. Assume in addition the following: $\forall t \in[0, T]$,

$$
\xi^{1} \leq \xi^{2}, f^{1}\left(t, Y_{t}^{1}, Z_{t}^{1}\right) \leq f^{2}\left(t, Y_{t}^{1}, Z_{t}^{1}\right), L_{t}^{1} \leq L_{t}^{2},
$$

then $Y_{t}^{1} \leq Y_{t}^{2}$, for $t \in[0, T]$. 
Proof. Applying Itô's formula to $\left[\left(Y^{1}-Y^{2}\right)^{+}\right]^{2}$ on interval $[t, T]$, and taking expectation on the both sides, since on the set $\left\{Y_{t}^{1}>Y_{t}^{2}\right\}, Y_{t}^{1}>Y_{t}^{2} \geq L_{t}^{2} \geq L_{t}^{1}$, we have

$$
\int_{t}^{T}\left(Y_{s}^{1}-Y_{s}^{2}\right)^{+} d\left(K_{s}^{1}-K_{s}^{2}\right)=-\int_{t}^{T}\left(Y_{s}^{1}-Y_{s}^{2}\right)^{+} d K_{s}^{2} \leq 0
$$

then we get immediately

$$
\begin{aligned}
& E\left[\left(Y_{t}^{1}-Y_{t}^{2}\right)^{+}\right]^{2}+E \int_{t}^{T} 1_{\left\{Y_{t}^{1}>Y_{t}^{2}\right\}}\left|Z_{s}^{1}-Z_{s}^{2}\right|^{2} d s \\
\leq & \frac{1}{2} E \int_{t}^{T} 1_{\left\{Y_{t}^{1}>Y_{t}^{2}\right\}}\left|Z_{s}^{1}-Z_{s}^{2}\right|^{2} d s+\left(2 \mu+4 k^{2}\right) E \int_{t}^{T}\left[\left(Y_{s}^{1}-Y_{s}^{2}\right)^{+}\right]^{2} d s,
\end{aligned}
$$

in view of (56) and the Lipschitz condition and monotonic condition of $f^{2}$. Hence

$$
E\left[\left(Y_{t}^{1}-Y_{t}^{2}\right)^{+}\right]^{2} \leq\left(2 \mu+4 K^{2}\right) E \int_{t}^{T}\left[\left(Y_{s}^{1}-Y_{s}^{2}\right)^{+}\right]^{2} d s
$$

from Gronwall's inequality, we deduce $\left(Y_{t}^{1}-Y_{t}^{2}\right)^{+}=0,0 \leq t \leq T$.

Then we prove a comparison theorem for the increasing processes under Lipschitz assumption on $f$ via the penalization method in [12].

Theorem 3.2 Suppose that the parameters $\left(\xi^{1}, f^{1}, L^{1}, U^{1}\right)$ and $\left(\xi^{2}, f^{2}, L^{2}, U^{2}\right)$ satisfy the following conditions: for $i=1,2$,

(i) $\xi^{i} \in \mathbf{L}^{2}\left(\mathcal{F}_{T}\right)$;

(ii) $f^{i}$ satisfy assumption 2.2-(i), (iii), (vi) and a Lipschitz condition in $(y, z)$ uniformly in $(t, \omega)$, i.e. there exists a constant $k$ such that, for $y, y^{\prime} \in \mathbb{R}, z, z^{\prime} \in \mathbb{R}^{d}$,

$$
\left|f^{i}(t, y, z)-f^{i}\left(t, y^{\prime}, z^{\prime}\right)\right| \leq k\left(\left|y-y^{\prime}\right|+\left|z-z^{\prime}\right|\right)
$$

(iii) $L^{i}$ and $U^{i}$ are real-valued, $\mathcal{F}_{t}$-adapted, continuous with $\left(L^{i}\right)^{+},\left(U^{i}\right)^{-} \in \mathbf{S}^{2}(0, T)$.

Let $\left(Y^{i}, Z^{i}, K^{i,+}, K^{i,-}\right)$ be the solution of the $\operatorname{RBSDE}\left(\xi^{i}, f^{i}, L^{i}, U^{i}\right)$, i.e.

$$
Y_{t}^{i}=\xi^{i}+\int_{t}^{T} f^{i}\left(s, Y_{s}^{i}, Z_{s}^{i}\right) d s+K_{T}^{i,+}-K_{t}^{i,+}-\left(K_{T}^{i,-}-K_{t}^{i,-}\right)-\int_{t}^{T} Z_{s}^{i} d B_{s},
$$

$Y_{t}^{i} \geq L_{t}^{i}, 0 \leq t \leq T$, and $\int_{0}^{T}\left(Y_{s}^{i}-L_{s}^{i}\right) d K_{s}^{i,+}=\int_{0}^{T}\left(Y_{s}^{i}-U_{s}^{i}\right) d K_{s}^{i,-}=0$,

Moreover, we assume $\forall(t, y, z) \in[0, T] \times \mathbb{R} \times \mathbb{R}^{d}$,

$$
\xi^{1} \leq \xi^{2}, f^{1}(t, y, z) \leq f^{2}(t, y, z),
$$

Then we have: for $0 \leq t \leq T$,

(i) If $L^{1}=L^{2}, U^{1}=U^{2}$, then $Y_{t}^{1} \leq Y_{t}^{2}, K_{t}^{1,+} \geq K_{t}^{2,+}, K_{t}^{1,-} \leq K_{t}^{2,-}$, and $d K^{1,+} \geq d K^{2,+}$, $d K^{1,-} \leq d K^{2,-}$;

(ii) If $L_{t}^{1} \leq L_{t}^{2}, U_{t}^{1}=U_{t}^{2}$, then $Y_{t}^{1} \leq Y_{t}^{2}, K_{t}^{1,-} \leq K_{t}^{2,-}$, and $d K^{1,-} \leq d K^{2,-}$;

(iii) If $L_{t}^{1}=L_{t}^{2}, U_{t}^{1} \leq U_{t}^{2}$, then $Y_{t}^{1} \leq Y_{t}^{2}, K_{t}^{1,+} \geq K_{t}^{2,+}$, and $d K^{1,+} \geq d K^{2,+}$.

Proof. (i) Set $L:=L^{1}=L^{2}, U:=U^{1}=U^{2}$, and consider the penalization equations for $m$, $n \in \mathbf{N}, i=1,2$

$$
Y_{t}^{m, n, i}=\xi^{i}+\int_{t}^{T} f^{i}\left(s, Y_{s}^{m, n, i}, Z_{s}^{m, n, i}\right) d s+m \int_{t}^{T}\left(Y_{s}^{m, n, i}-L_{s}\right)^{-} d s-n \int_{t}^{T}\left(Y_{s}^{m, n, i}-U_{s}\right)^{+} d s-\int_{t}^{T} Z_{s}^{m, n, i} d B_{s} .
$$


By comparison theorem for BSDEs, since $\xi^{1} \leq \xi^{2}$ and

$$
f^{1}(t, y, z)+m\left(y-L_{t}\right)^{-}-n\left(y-U_{t}\right)^{+} \leq f^{2}(t, y, z)+m\left(y-L_{t}\right)^{-}-n\left(y-U_{t}\right)^{+},
$$

we have $Y_{t}^{m, n, 1} \leq Y_{t}^{m, n, 2}, \forall t \in[0, T]$. Denote $K_{t}^{m, n, i,+}=m \int_{0}^{t}\left(Y_{s}^{m, n, i}-L_{s}\right)^{-} d s$, then for $0 \leq s \leq$ $t \leq T$,

$$
K_{t}^{m, n, 1,+}-K_{s}^{m, n, 1,+} \geq K_{t}^{m, n, 2,+}-K_{s}^{m, n, 2,+} .
$$

From the convergence results in [12], which also holds for Lipschitz function,

$$
\lim _{n \rightarrow \infty} \lim _{m \rightarrow \infty} Y_{t}^{m, n, i}=Y_{t}^{i}, \lim _{n \rightarrow \infty} \lim _{m \rightarrow \infty} K_{t}^{m, n, i,+}=K_{t}^{i,+},
$$

in $\mathbf{L}^{2}\left(\mathcal{F}_{t}\right)$, where $Y^{i}, K^{i,+}, K^{i,-}$ are elements of the solution of $\operatorname{RBSDE}\left(\xi^{i}, f^{i}, L, U\right)$. Consequently, for $0 \leq s \leq t \leq T$,

$$
Y_{t}^{1} \leq Y_{t}^{2}, K_{t}^{1,+}-K_{s}^{1,+} \geq K_{t}^{2,+}-K_{s}^{2,+}
$$

if we especially set $s=0$, we get $K_{t}^{1,+} \geq K_{t}^{2,+}$. Similarly $K_{t}^{1,-} \leq K_{t}^{2,-}$.

(ii) Set $U:=U^{1}=U^{2}$, we consider the penalized reflected BSDE's, for $n \in \mathbf{N}, i=1,2$,

$$
\begin{aligned}
& Y_{t}^{n, i}=\xi^{i}+\int_{t}^{T} f^{i}\left(s, Y_{s}^{n, i}, Z_{s}^{n, i}\right) d s+K_{T}^{n,+}-K_{t}^{n,+}-n \int_{t}^{T}\left(Y_{s}^{n, i}-U_{s}\right)^{+} d s-\int_{t}^{T} Z_{s}^{n, i} d B_{s}, \\
& Y_{t}^{n, i} \geq L_{t}^{i}, \int_{0}^{T}\left(Y_{t}^{n, i}-L_{t}^{i}\right) d K_{t}^{n,+}=0 .
\end{aligned}
$$

Since $\forall t \in[0, T]$,

$$
\xi^{1} \leq \xi^{2}, \quad f^{1}(t, y, z)-n\left(y-U_{t}\right)^{+} \leq f^{2}(t, y, z)-n\left(y-U_{t}\right)^{+}, \quad L_{t}^{1} \leq L_{t}^{2}
$$

by the comparison theorem for RBSDE with one barrier, we have $Y_{t}^{n, 1} \leq Y_{t}^{n, 2}$. Let $K_{t}^{n, i,-}=$ $n \int_{0}^{t}\left(Y_{s}^{n, i}-U_{s}\right)^{+} d s$, then for $0 \leq s \leq t \leq T$,

$$
K_{t}^{n, 1,-}-K_{s}^{n, 1,-} \leq K_{t}^{n, 2,-}-K_{s}^{n, 2,-} .
$$

Thanks to the convergence result in [12, which still works for Lipschitz functions, we have for $0 \leq s \leq t \leq T$,

$$
Y_{t}^{1} \leq Y_{t}^{2}, K_{t}^{1,-}-K_{s}^{1,-} \leq K_{t}^{2,-}-K_{s}^{2,-}
$$

if we especially set $s=0$, we get $K_{t}^{1,+} \geq K_{t}^{2,+}, K_{t}^{1,-} \leq K_{t}^{2,-}$.

(iii) The proof is in the same as (ii), so we omit it.

We next prove a comparison theorem for RBSDE with two barriers in a general case.

Theorem 3.3 (General case for RBSDE's) Suppose that the parameters $\left(\xi^{1}, f^{1}, L^{1}, U^{1}\right)$ and $\left(\xi^{2}, f^{2}, L^{2}, U^{2}\right)$ satisfy assumption 2.1, 2.2 and 2.3. Let $\left(Y^{1}, Z^{1}, K^{1,+}, K^{1,-}\right),\left(Y^{2}, Z^{2}, K^{2,+}, K^{2,-}\right)$ be respectively the solutions of the $R B S D E\left(\xi^{1}, f^{1}, L^{1}, U^{1}\right)$ and $R B S D E\left(\xi^{2}, f^{2}, L^{2}, U^{2}\right)$ as definition 2.1. Assume in addition the following: $\forall t \in[0, T]$

$$
\begin{aligned}
& \xi^{1} \leq \xi^{2}, \quad f^{1}\left(t, Y_{t}^{1}, Z_{t}^{1}\right) \leq f^{2}\left(t, Y_{t}^{1}, Z_{t}^{1}\right), \\
& L_{t}^{1} \leq L_{t}^{2}, \quad U_{t}^{1} \leq U_{t}^{2},
\end{aligned}
$$

then $Y_{t}^{1} \leq Y_{t}^{2}$, for $t \in[0, T]$. 
Proof. Applying Ito's formula to $\left[\left(Y^{1}-Y^{2}\right)^{+}\right]^{2}$ on the interval $[t, T]$, and taking expectation on the both sides, we get immediately

$$
\begin{aligned}
& E\left[\left(Y_{t}^{1}-Y_{t}^{2}\right)^{+}\right]^{2}+E \int_{t}^{T} 1_{\left\{Y_{t}^{1}>Y_{t}^{2}\right\}}\left|Z_{s}^{1}-Z_{s}^{2}\right|^{2} d s \\
\leq & 2 E \int_{t}^{T} 1_{\left\{Y_{s}^{1}>Y_{s}^{2}\right\}}\left(Y_{s}^{1}-Y_{s}^{2}\right)\left(f^{2}\left(s, Y_{s}^{1}, Z_{s}^{1}\right)-f^{2}\left(s, Y_{s}^{2}, Z_{s}^{2}\right)\right) d s \\
\leq & \frac{1}{2} E \int_{t}^{T} 1_{\left\{Y_{t}^{1}>Y_{t}^{2}\right\}}\left|Z_{s}^{1}-Z_{s}^{2}\right|^{2} d s+\left(2 \mu+4 k^{2}\right) E \int_{t}^{T}\left[\left(Y_{s}^{1}-Y_{s}^{2}\right)^{+}\right]^{2} d s
\end{aligned}
$$

in view of (58) and the Lipschitz condition and monotonic condition on $f^{2}$, and the fact that

$$
\int_{t}^{T}\left(Y_{s}^{1}-Y_{s}^{2}\right)^{+} d\left(K_{s}^{1,+}-K_{s}^{2,+}\right) \leq 0, \int_{t}^{T}\left(Y_{s}^{1}-Y_{s}^{2}\right)^{+} d\left(K_{s}^{1,-}-K_{s}^{2,-}\right) \geq 0,
$$

which is similar to reflected BSDE with one barrier. Hence

$$
E\left[\left(Y_{t}^{1}-Y_{t}^{2}\right)^{+}\right]^{2} \leq\left(2 \mu+4 k^{2}\right) E \int_{t}^{T}\left[\left(Y_{s}^{1}-Y_{s}^{2}\right)^{+}\right]^{2} d s
$$

and from Gronwall's inequality, we deduce $\left(Y_{t}^{1}-Y_{t}^{2}\right)^{+}=0,0 \leq t \leq T$.

From the convergence of penalization equations, we get the following comparison theorem.

Theorem 3.4 (Special case) Suppose that $f^{1}(s, y), f^{2}(s, y)$ satisfy assumption 2.4, and $\xi^{i}, f^{i}(\cdot, 0)$, $L, U, i=1,2$ satisfies the bounded assumption (3). The two triples $\left(Y^{1}, Z^{1}, K^{1}\right),\left(Y^{2}, Z^{2}, K^{2}\right)$ are respectively the solutions of the $R B S D E\left(\xi^{1}, f^{1}, L, U\right)$ and $R B S D E\left(\xi^{2}, f^{2}, L, U\right)$ as definition 2.1. If we have

$$
\xi^{1} \leq \xi^{2}, \text { and } f^{1}(t, y) \leq f^{2}(t, y), \forall(t, y) \in[0, T] \times \mathbb{R}
$$

then $Y_{t}^{1} \leq Y_{t}^{2}, K_{t}^{1,+} \geq K_{t}^{2,+}, K_{t}^{1,-} \leq K_{t}^{2,-}$, for $t \in[0, T]$, and $d K^{1,+} \geq d K^{2,+}, d K^{1,-} \leq d K^{2,-}$.

Proof. We consider the penalized equations relative to the $\operatorname{RBSDE}\left(\xi^{i}, f^{i}, L, U\right)$, for $i=1,2$, $n \in \mathbf{N}$,

$Y_{t}^{m, n, i}=\xi^{i}+\int_{t}^{T} f^{i}\left(s, Y_{s}^{m, n, i}\right) d s+n \int_{t}^{T}\left(Y_{s}^{m, n, i}-L_{s}\right)^{-} d s-m \int_{t}^{T}\left(Y_{s}^{m, n, i}-U_{s}\right)^{+} d s-\int_{t}^{T} Z_{s}^{m, n, i} d B_{s}$.

For each $m, n \in \mathbf{N}$,

$f^{m, n, 1}(s, y)=f^{1}(s, y)+n\left(y-L_{s}\right)^{-}-m\left(y-U_{s}\right)^{+} \leq f^{m, n, 2}(s, y)=f^{2}(s, y)+n\left(y-L_{s}\right)^{-}-m\left(y-U_{s}\right)^{+}$,

and $\xi^{1} \leq \xi^{2}$. So by the comparison theorem in [13], we get

$$
Y_{t}^{m, n, 1} \leq Y_{t}^{m, n, 2}, 0 \leq t \leq T .
$$

Since $K_{t}^{m, n, i,+}=n \int_{0}^{t}\left(Y_{s}^{m, n, i}-L_{s}\right)^{-} d s$, then we deduce, for $0 \leq s \leq t \leq T$,

$$
K_{t}^{m, n, 1,+}-K_{s}^{m, n, 1,+} \geq K_{t}^{m, n, 2,+}-K_{s}^{m, n, 2,+},
$$

By the convergence results of the step1, we know tha the inequalities hold for $0 \leq s \leq t \leq T$ :

$$
Y_{t}^{1} \leq Y_{t}^{2}, K_{t}^{1,+}-K_{s}^{1,+} \geq K_{t}^{2,+}-K_{s}^{2,+},
$$

Particularly, set $s=0$, we get $K_{t}^{1,+} \geq K_{t}^{2,+}$. Symmetrically, $K_{t}^{1,-} \leq K_{t}^{2,-}$. 
Corollary 3.1 Suppose that $f^{1}(s, y), f^{2}(s, y)$ satisfy assumption 2.4, and $\xi^{i}, f^{i}(\cdot, 0), L^{i}, U^{i}, i=$ 1,2 satisfies (3). The two triples $\left(Y^{1}, Z^{1}, K^{1}\right),\left(Y^{2}, Z^{2}, K^{2}\right)$ are respectively the solutions of the $\operatorname{RBSDE}\left(\xi^{1}, f^{1}, L^{1}, U^{1}\right)$ and $\operatorname{RBSDE}\left(\xi^{2}, f^{2}, L^{2}, U^{2}\right)$, with $K^{i}=K^{i,+}-K^{i,-}, i=1,2$. In addition, we assume

$$
\xi^{1} \leq \xi^{2}, \text { and } f^{1}(t, y) \leq f^{2}(t, y), \forall(t, y) \in[0, T] \times \mathbb{R},
$$

then for $0 \leq t \leq T$, (i) If $L_{t}^{1} \leq L_{t}^{2}, U_{t}^{1}=U_{t}^{2}$, then $Y_{t}^{1} \leq Y_{t}^{2}, K_{t}^{1,-} \leq K_{t}^{2,-}$, and $d K^{1,-} \leq d K^{2,-}$;

(ii) If $L_{t}^{1}=L_{t}^{2}, U_{t}^{1} \leq U_{t}^{2}$, then $Y_{t}^{1} \leq Y_{t}^{2}, K_{t}^{1,+} \geq K_{t}^{2,+}$, and $d K^{1,+} \geq d K^{2,+}$.

Proof. (i) To simplify symbols, we denote $U=U^{1}=U^{2}$. For $n \in \mathbf{N}$, we consider the following RBSDE with one barrier $L^{i}, i=1,2$.

$$
\begin{aligned}
& Y_{t}^{n, i}=\xi^{i}+\int_{t}^{T} f^{i}\left(s, Y_{s}^{n, i}\right) d s+K_{T}^{n, i,+}-K_{t}^{n, i,+}-n \int_{t}^{T}\left(Y_{s}^{n, i}-U_{s}\right)^{+} d s-\int_{t}^{T} Z_{s}^{n, i} d B_{s}, \\
& Y_{t}^{n, i} \geq L_{t}^{i}, \int_{0}^{T}\left(Y_{t}^{n, i}-L_{t}^{i}\right) d t=0 .
\end{aligned}
$$

Since $\xi^{1} \leq \xi^{2}, f^{1}(t, y) \leq f^{2}(t, y), L_{t}^{1} \leq L_{t}^{2}$, by general comparison theorem of RBSDE with one barrier, we know $Y_{t}^{n, 1} \leq Y_{t}^{n, 2}$. Denote $K_{t}^{n, 1,-}=n \int_{0}^{t}\left(Y_{s}^{n, 1}-U_{s}\right)^{+} d s, K_{t}^{n, 2,-}=n \int_{0}^{t}\left(Y_{s}^{n, 2}-U_{s}\right)^{+} d s$, then for $0 \leq s \leq t \leq T$,

$$
K_{t}^{n, 1,-}-K_{s}^{n, 1,-} \leq K_{t}^{n, 2,-}-K_{s}^{n, 2,-} .
$$

Thanks to the convergence result of step 1of theorem 2.2, it follows immediately that for $0 \leq s \leq$ $t \leq T$

$$
Y_{t}^{1} \leq Y_{t}^{2} \text { and } K_{t}^{1,-}-K_{s}^{1,-} \leq K_{t}^{2,-}-K_{s}^{2,-} .
$$

Especially with $s=0$, we get $K_{t}^{1,-} \leq K_{t}^{2,-}$.

(ii) follows similarly as (i), so we omit it

Theorem 3.5 Suppose that $f^{1}(s, y), f^{2}(s, y)$ satisfy assumption $2.4, \xi^{i}, f^{i}(\cdot, 0), U^{i}$, for $i=1,2$ satisfies (21), and $L^{i}$ satisfies assumption 2.3'. The two groups $\left(Y^{1}, Z^{1}, K^{1}\right),\left(Y^{2}, Z^{2}, K^{2}\right)$ are respectively the solutions of the $\operatorname{RBSDE}\left(\xi^{1}, f^{1}, L^{1}, U^{1}\right)$ and $R B S D E\left(\xi^{2}, f^{2}, L^{2}, U^{2}\right)$, as definition 2.1. Moreover, assume

$$
\xi^{1} \leq \xi^{2}, f^{1}(t, y) \leq f^{2}(t, y), \forall(t, y) \in[0, T] \times \mathbb{R}
$$

then for $0 \leq t \leq T$,

(i) If $L^{1}=L^{2}$ and $U^{1}=U^{2}$, then $Y_{t}^{1} \leq Y_{t}^{2}, K_{t}^{1,+} \geq K_{t}^{2,+}, K_{t}^{1,-} \leq K_{t}^{2,-}$, and $d K^{1,+} \geq d K^{2,+}$, $d K^{1,-} \leq d K^{2,-}$;

(ii) If $L_{t}^{1} \leq L_{t}^{2}, U_{t}^{1}=U_{t}^{2}$, then $Y_{t}^{1} \leq Y_{t}^{2}, K_{t}^{1,-} \leq K_{t}^{2,-}$, and $d K^{1,-} \leq d K^{2,-}$;

(iii) If $L_{t}^{1}=L_{t}^{2}, U_{t}^{1} \leq U_{t}^{2}$, then $Y_{t}^{1} \leq Y_{t}^{2}, K_{t}^{1,+} \geq K_{t}^{2,+}$, and $d K^{1,+} \geq d K^{2,+}$.

Proof. Like in the step 2 of the proof of theorem 2.2, we approximate the barrier $L^{i}$ by super bounded barrier $L^{n, i}$, where $L^{n, i}=L^{i} \wedge n$.

(i) Set $L:=L^{1}=L^{2}, U:=U^{1}=U^{2}$, and $L^{n}=L \wedge n$. Then consider the $\operatorname{RBSDE}\left(\xi^{i}, f^{i}, L^{n}, U\right)$, for $i=1,2$,

$$
\begin{aligned}
Y_{t}^{n, i} & =\xi^{i}+\int_{t}^{T} f^{i}\left(s, Y_{s}^{n, i}\right) d s+K_{T}^{n, i,+}-K_{t}^{n, i,+}-\left(K_{T}^{n, i,-}-K_{t}^{n, i,-}\right)-\int_{t}^{T} Z_{s}^{n, i} d B_{s}, \\
L_{t}^{n} & \leq Y_{t}^{n, i} \leq U_{t}, \int_{0}^{T}\left(Y_{s}^{n, i}-L_{s}\right) d K_{s}^{n, i,+}=\int_{0}^{T}\left(Y_{s}^{n, i}-U_{s}\right) d K_{s}^{n, i,-}=0 .
\end{aligned}
$$


Since

$$
\xi^{1} \leq \xi^{2}, f^{1}(t, y) \leq f^{2}(t, y),
$$

from comparison theorem 3.4, we have for $0 \leq s \leq t \leq T$,

$$
Y_{t}^{n, 1} \leq Y_{t}^{n, 2}, K_{t}^{n, 1,+}-K_{s}^{n, 1,+} \geq K_{t}^{n, 2,+}-K_{s}^{n, 2,+} .
$$

Thanks to the convergence results in step 2 of the proof for theorem 2.2, we get that

$$
Y_{t}^{1} \leq Y_{t}^{2}, K_{t}^{1,+}-K_{s}^{1,+} \geq K_{t}^{2,+}-K_{s}^{2,+} \text {, for } 0 \leq s \leq t \leq T .
$$

Especially, with $s=0$, we get $K_{t}^{1,+} \geq K_{t}^{2,+}$. Similarly $K_{t}^{1,-} \leq K_{t}^{2,-}$, for $t \in[0, T]$.

(ii) Set $U:=U^{1}=U^{2}$, and $L^{n, i}=L^{i} \wedge n$. Then we consider the solutions $\left(Y^{n, i}, Z^{n, i}, K^{n, i}\right)$ of the RBSDEs $\left(\xi^{i}, f^{i}, L^{n, i}, U\right)$, for $i=1,2$. Since

$$
\xi^{1} \leq \xi^{2}, f^{1}(t, y) \leq f^{2}(t, y), L_{t}^{n, 1} \leq L_{t}^{n, 2},
$$

from corollary 3.1, we have for $0 \leq s \leq t \leq T, Y_{t}^{n, 1} \leq Y_{t}^{n, 2}$ and $K_{t}^{n, 1,-}-K_{s}^{n, 1,-} \leq K_{t}^{n, 2,-}-K_{s}^{n, 2,-}$. Then by the convergence results in step 2 of the proof for theorem 2.2 , it follows that

$$
Y_{t}^{1} \leq Y_{t}^{2}, K_{t}^{1,-}-K_{s}^{1,-} \leq K_{t}^{2,-}-K_{s}^{2,-} \text {, for } 0 \leq s \leq t \leq T, .
$$

Especially, with $s=0$, we get $K_{t}^{1,-} \leq K_{t}^{2,-}$, for $t \in[0, T]$.

(iii) The proof is similar to (ii), which follows from corollary 3.1 and by the convergence results in step 2 of the proof for theorem [2.2, so we omit it.

Theorem 3.6 Suppose that $f^{1}(s, y), f^{2}(s, y)$ satisfy assumption $2.4, \xi^{i}, f^{i}(\cdot, 0)$, for $i=1,2$ satisfies the bounded assumption (37), and $L^{i}$ and $U^{i}$ satisfy assumption 2.3'. The two groups $\left(Y^{1}, Z^{1}, K^{1}\right),\left(Y^{2}, Z^{2}, K^{2}\right)$ are respectively the solutions of the $R B S D E\left(\xi^{1}, f^{1}, L^{1}, U^{1}\right)$ and $R B S D E\left(\xi^{2}, f^{2}, L^{2}, U^{2}\right)$.

Moreover, assume

$$
\xi^{1} \leq \xi^{2}, f^{1}(t, y) \leq f^{2}(t, y), \forall(t, y) \in[0, T] \times \mathbb{R},
$$

then for $t \in[0, T]$,

(i) If $L^{1}=L^{2}$ and $U^{1}=U^{2}$, then $Y_{t}^{1} \leq Y_{t}^{2}, K_{t}^{1,+} \geq K_{t}^{2,+}, K_{t}^{1,-} \leq K_{t}^{2,-}$, and $d K^{1,+} \geq d K^{2,+}$, $d K^{1,-} \leq d K^{2,-}$;

(ii) If $L_{t}^{1} \leq L_{t}^{2}, U_{t}^{1}=U_{t}^{2}$, then $Y_{t}^{1} \leq Y_{t}^{2}, K_{t}^{1,-} \leq K_{t}^{2,-}$, and $d K^{1,-} \leq d K^{2,-}$;

(iii) If $L_{t}^{1}=L_{t}^{2}, U_{t}^{1} \leq U_{t}^{2}$, then $Y_{t}^{1} \leq Y_{t}^{2}, K_{t}^{1,+} \geq K_{t}^{2,+}$, and $d K^{1,+} \geq d K^{2,+}$.

Proof. Like in theorem 3.5, we approximate the barrier $U$ by lower bounded barrier $U^{n}$, where $U^{n}=U \vee(-n)$, then the results follow from the comparison theorem 3.5 and the convergence results of step 3 in the proof of theorem 2.2 , so we omit it.

Theorem 3.7 Suppose that for $i=1,2$, $\xi^{i}$ satisfies assumption 2.1, $f^{i}$ does not depends on $z$ and satisfies assumption 2.2, $L^{i}$ and $U^{i}$ satisfy assumption 2.3. The two triples $\left(Y^{1}, Z^{1}, K^{1,+}, K^{1,-}\right)$, $\left(Y^{2}, Z^{2}, K^{2,+}, K^{2,-}\right)$ are the solutions of the $\operatorname{RBSDE}\left(\xi^{1}, f^{1}, L^{1}, U^{1}\right)$ and $\operatorname{RBSDE}\left(\xi^{2}, f^{2}, L^{2}, U^{2}\right)$, respectively. Moreover, assume for $(t, y) \in[0, T] \times \mathbb{R}$,

$$
\xi^{1} \leq \xi^{2}, f^{1}(t, y) \leq f^{2}(t, y), \text { and } f^{1}(t, 0)=f^{2}(t, 0),
$$

then for $t \in[0, T]$,

(i) If $L^{1}=L^{2}$ and $U^{1}=U^{2}$, then $Y_{t}^{1} \leq Y_{t}^{2}, K_{t}^{1,+} \geq K_{t}^{2,+}, K_{t}^{1,-} \leq K_{t}^{2,-}$, and $d K^{1,+} \geq d K^{2,+}$, $d K^{1,-} \leq d K^{2,-}$;

(ii) If $L_{t}^{1} \leq L_{t}^{2}, U_{t}^{1}=U_{t}^{2}$, then $Y_{t}^{1} \leq Y_{t}^{2}, K_{t}^{1,-} \leq K_{t}^{2,-}$, and for $d K^{1,-} \leq d K^{2,-}$;

(iii) If $L_{t}^{1}=L_{t}^{2}, U_{t}^{1} \leq U_{t}^{2}$, then $Y_{t}^{1} \leq Y_{t}^{2}, K_{t}^{1,+} \geq K_{t}^{2,+}$, and for $d K^{1,+} \geq d K^{2,+}$. 
Proof. (i)Set $L:=L^{1}=L^{2}, U:=U^{1}=U^{2}$. Like in the proof of the theorem 2.2, for $i=1,2$, set

$$
\left(\bar{Y}_{t}^{i}, \bar{Z}_{t}^{i}, \bar{K}_{t}^{i,+}, \bar{K}_{t}^{i,-}\right):=\left(e^{\lambda t} Y_{t}^{i}, e^{\lambda t} Z_{t}^{i}, \int_{0}^{t} e^{\lambda s} d K_{s}^{i,+}, \int_{0}^{t} e^{\lambda s} d K_{s}^{i,-}\right) .
$$

Then it's easy to check that for $i=1,2,\left(\bar{Y}_{t}^{i}, \bar{Z}_{t}^{i}, \bar{K}_{t}^{i,+}, \bar{K}_{t}^{i,-}\right)_{0 \leq t \leq T}$ is the solution of the $\operatorname{RBSDE}\left(\bar{\xi}^{i}, \bar{f}^{i}, \bar{L}, \bar{U}\right)$, where

$$
\left(\bar{\xi}^{i}, \bar{f}^{i}(t, y), \bar{L}_{t}, \bar{U}_{t}\right)=\left(e^{\lambda T} \xi^{i}, e^{\lambda t} f^{i}\left(t, e^{-\lambda t} y\right)-\lambda y, e^{\lambda t} L_{t}, e^{\lambda t} U_{t}\right) .
$$

If we assume $\lambda=\mu$, then $\left(\bar{\xi}^{i}, \bar{f}^{i}, \bar{L}, \bar{U}\right)$ satisfies assumption 2.1, 2.4 and 2.3'. Since the transform keeps the monotonicity, the results are equivalent to

$$
\bar{Y}_{t}^{1} \leq \bar{Y}_{t}^{2}, \bar{K}_{t}^{1,+}-\bar{K}_{s}^{1,+} \geq \bar{K}_{t}^{2,+}-\bar{K}_{s}^{2,+}, \bar{K}_{t}^{1,-}-\bar{K}_{s}^{1,-} \geq \bar{K}_{t}^{2,-}-\bar{K}_{s}^{2,-},
$$

for $0 \leq s \leq t \leq T$. Then we make the approximations

$$
\begin{aligned}
\bar{\xi}^{m, n, i}: & =\bar{\xi}^{n, i} \wedge m:=\left(\bar{\xi}^{i} \vee(-n)\right) \wedge m \\
\bar{f}_{m, n}^{i}(t, y): & =\bar{f}_{n}^{i}(t, y)-\bar{f}_{n}^{i}(t, 0)+\bar{f}_{n}^{i}(t, 0) \wedge m \\
: & =\bar{f}^{i}(t, y)-\bar{f}^{i}(t, 0)+\left(\bar{f}^{i}(t, 0) \vee(-n)\right) \wedge m
\end{aligned}
$$

Let for $i=1,2,\left(\bar{Y}_{t}^{m, n, i}, \bar{Z}_{t}^{m, n, i}, \bar{K}_{t}^{m, n, i,+}, \bar{K}_{t}^{m, n, i,-}\right)_{0 \leq t \leq T}$ be the solution of the RBSDE $\left(\bar{\xi}^{m, n, i}, \bar{f}_{m, n}^{i}, \bar{L}, \bar{U}\right)$; then $\bar{\xi}^{m, n, i}, \bar{f}_{m, n}^{i}$ satisfy

$$
\left|\bar{\xi}^{m, n, i}\right|+\sup _{0 \leq t \leq T}\left|\bar{f}_{m, n}^{i}(t, 0)\right| \leq c
$$

and

$$
\bar{\xi}^{m, n, 1} \leq \bar{\xi}^{m, n, 2}, \text { and } \bar{f}_{m, n}^{1}(t, y) \leq \bar{f}_{m, n}^{2}(t, y), \text { for }(t, y) \in[0, T] \times \mathbb{R},
$$

in view of $\bar{f}^{1}(t, 0)=f^{1}(t, 0)=f^{2}(t, 0)=\bar{f}^{2}(t, 0)$. Using the comparison theorem 3.6-(i), we have for $0 \leq s \leq t \leq T$

$$
\bar{Y}_{t}^{m, n, 1} \leq \bar{Y}_{t}^{m, n, 2}, \bar{K}_{t}^{m, n, 1,+}-\bar{K}_{s}^{m, n, 1,+} \geq \bar{K}_{t}^{m, n, 2,+}-\bar{K}_{s}^{m, n, 2,+} .
$$

By the convergence results in the step 4 and step 5 of the proof of theorem 2.2 , let $m \rightarrow \infty$, then $n \rightarrow \infty$, we get for $0 \leq s \leq t \leq T$

$$
\bar{Y}_{t}^{1} \leq \bar{Y}_{t}^{2}, \bar{K}_{t}^{1,+}-\bar{K}_{s}^{1,+} \geq \bar{K}_{t}^{2,+}-\bar{K}_{s}^{2,+}
$$

Especially, with $s=0$, it follows $\bar{K}_{t}^{1,+} \geq \bar{K}_{t}^{2,+}$. Similarly $\bar{K}_{t}^{1,-} \leq \bar{K}_{t}^{2,-}$.

(ii) and (iii) are from comparison theorem 3.6-(ii) and (iii), with approximation as in (i), so we omit it. $\square$

Acknowledgement Author thanks professor Jean-Pierre Lepeltier for his helps when author worked on this paper.

\section{References}

[1] Alario Nazaret M., Lepeltier, J.P. and Marchal. B., 1982. Dynkin games. Lecture Notes in Control and Inform. Sci. 43. (Springer, Berlin), 23-42.

[2] Bismut, J.M., 1977. Sur un problème de Dynkin. Z.Wahrsch. Verw. Gebiete 39 31-53. 
[3] Briand, Ph., Delyon, B., Hu, Y., Pardoux, E. and Stoica, L. (2003) $L_{p}$ solutions of BSDEs, Stochastic Process. Appl. 108, 109-129.

[4] Cvitanic, J. and Karatzas, I., 1996. Backward Stochastic Differential Equations with Reflection and Dykin Games, Ann. Probab. 24 , no 4, 2024-2056.

[5] Elliott, R., 1976. The existence of value in stochastic differential games. SIAM JCO 14(1), 85-94.

[6] El Karoui, N., Kapoudjian, C., Pardoux, E., Peng, S. and Quenez, M.C., 1997. Reflected Solutions of Backward SDE and Related Obstacle Problems for PDEs, Ann. Probab. 25, no $2,702-737$.

[7] El Karoui, N., Peng, S. and Quenez, M.C., 1997. Backward stochastic differential equations in Finance. Math. Finance, 7, 1-71.

[8] Hamadène, S., Lepeltier, J.P. and Matoussi, A. 1997. Double barrier backward SDEs with continuous coefficient. In: El Karoui, N. and Mazliak, L., (Eds.), Backward Stochastic differential Equaitons. Pitman Research Notes in Mathematics Series 364, pp. 161-177.

[9] Hamadène, S., Lepeltier, J.P. and Peng, S., 1997. BSDE with continuous coefficients and stochastic differential games. In: El Karoui, N. and Mazliak, L., (Eds.), Backward Stochastic differential Equaitons. Pitman Research Notes in Mathematics Series 364, pp. 115-128.

[10] Lepeltier, J.P. and Maingueneau, M.A., 1984. Le jeu de Dynkin en théorie générale sans l'hypothèse de Mokoboski. Stochstics. 13, 25-44.

[11] Lepeltier, J.P., Matoussi, A. and Xu, M. (2005) Reflected Backward stochastic differential equations under monotonicity and general increasing growth conditions. Advanced Applied Probability, 37, 1-26.

[12] Lepeltier, J.P. and San Martín, J., 2004. Backward SDE's with two barriers and continuous coefficient. An existence result. Journal of Applied Probability, vol. 41, 162-175.

[13] Pardoux, E., 1999. BSDE's, weak convergence and homogenization of semilinear PDE's in Nonlinear analysis, Differential Equations and Control, F. H. Clarke \& R. J. Stern Eds, pp. 503-549, Kluwer Acad. Pub.

[14] Pardoux, E. and Peng, S., 1990. Adapted solutions of Backward Stochastic Differential Equations. Systems Control Lett. 14, 51-61.

[15] Revuz, D. and Yor, M., 1991. Continuous martingales and Brownian motion. Springer, New York. 